\title{
Alginate-nanohydroxyapatite hydrogel system: Optimizing the formulation for enhanced bone regeneration
}

\author{
J. Barros ${ }^{\mathrm{a}, \mathrm{b}, \mathrm{c}, *}$, M.P. Ferraz ${ }^{\mathrm{d}}$, J. Azeredo ${ }^{\mathrm{e}}$, M.H. Fernandes ${ }^{\mathrm{f}, g}$, P.S. Gomes ${ }^{\mathrm{f}, \mathrm{g}}$, F.J. Monteiro ${ }^{\mathrm{a}, \mathrm{b}, \mathrm{c}}$ \\ ${ }^{a}$ i3S - Instituto de Investigação e Inovação em Saúde, Universidade do Porto, Porto, Portugal \\ ${ }^{\mathrm{b}}$ INEB - Instituto de Engenharia Biomédica, Universidade Porto, Porto, Portugal \\ ${ }^{\mathrm{c}}$ FEUP - Faculdade de Engenharia, DEMM, Universidade do Porto, Porto, Portugal \\ ${ }^{\mathrm{d}}$ FP-ENAS/CEBIMED - University Fernando Pessoa Energy, Environment and Health Research Unit/Biomedical Research Center, Porto, Portugal \\ ${ }^{\mathrm{e}}$ Laboratório de Investigação em Biofilmes Rosário Oliveira, Center of Biological Engineering, University of Minho, Braga, Portugal \\ ${ }^{\mathrm{f}}$ Laboratory for Bone Metabolism and Regeneration - Faculty of Dental Medicine, U. Porto, Porto, Portugal \\ ${ }^{8}$ LAQV/REQUIMTE, U. Port, Porto, Portugal
}

\section{A R T I C L E I N F O}

\section{Keywords:}

Biomaterials

Nanohydroxyapatite

Alginate

Hydrogel

Composite

Solubility

Biocompatibility

Osteogenic activity

\begin{abstract}
A B S T R A C T
Ceramic/polymer-based biocomposites have emerged as potential biomaterials to fill, replace, repair or regenerate injured or diseased bone, due to their outstanding features in terms of biocompatibility, bioactivity, injectability, and biodegradability. However, these properties can be dependent on the amount of ceramic component present in the polymer-based composite. Therefore, in the present study, the influence of nanohydroxyapatite content (30 to $70 \mathrm{wt} \%$ ) on alginate-based hydrogels was studied in order to evaluate the best formulation for maximizing bone tissue regeneration. The composite system was characterized in terms of physic-chemical properties and biological response, with in vitro cytocompatibility assessment with human osteoblastic cells and ex vivo functional evaluation in embryonic chick segmental bone defects. The main morphological characteristics of the alginate network were not affected by the addition of nanohydroxyapatite. However, physic-chemical features, like water-swelling rate, stability at extreme $\mathrm{pH}$ values, apatite formation, and $\mathrm{Ca}^{2+}$ release were nanoHA dose-dependent. Within in vitro cytocompatibility assays it was observed that hydrogels with nanoHA 30\% content enhanced osteoblastic cells proliferation and expression of osteogenic transcription factors, while those with higher concentrations (50 and 70\%) decreased the osteogenic cell response. Ex vivo data underlined the in vitro findings, revealing an enhanced collagenous deposition, trabecular bone formation and matrix mineralization with Alg-nanoHA30 composition, while compositions with higher nanoHA content induced a diminished bone tissue response.

The outcomes of this study indicate that nanohydroxyapatite concentration plays a major role in physicchemical properties and biological response of the composite system and the optimization of the components ratio must be met to maximize bone tissue regeneration.
\end{abstract}

*Corresponding author.

\section{Introduction}

Bone is a complex and dynamic tissue, which has the capability of remodeling and regenerating, up to a certain extent [1]. However, these physiological processes that contribute to tissue homeostasis can be impaired due to osteoporosis, rheumatoid arthritis, vitamin D deficiency and other metabolic conditions or traumatic fractures. These conditions have a huge impact on diminishing patients' quality of life and strongly affect healthcare costs, owing to requirements for surgeries, long-term hospitalization and difficult recoveries [1-4]. Materials like auto-/allografts, inert metallic and ceramic implants have been used to fill, replace, repair or regenerate injured or diseased bone with clinical success [1-3]. However, these applications have shown some associated limitations such as the risk of long-term foreign body reaction, pathogen transmission, immunogenic rejection, impaired bone formation, poor vascularization and integration, and inaccurate fitting to the defect size and shape [2-5].

Composites, based on the combination of different materials, such as polymers and ceramics, have emerged, aiming to solve these limitations in bone tissue engineering applications [6]. They can be

\footnotetext{
* Corresponding author.

E-mail address: joana.barros@ineb.up.pt (J. Barros).
} 
designed to adjust to bone defects' shape and geometry, occupying the available space in the damaged organ/tissue, precluding invagination of the neighboring tissues and priming tissue healing [2,6]. Furthermore, they can easily be modified or co-formulated with other components which impart additional properties such as osteoconduction, osteoinduction and osteogenicity [2].

Among polymers, polysaccharides are very versatile, enabling signaling molecules loading (e.g. growth factors, biomodulators, drugs) and an intrinsic interaction with inorganic components [7]. In this regard, alginate is a $\mathrm{pH}$-sensitive, natural, hydrophilic, biocompatible, injectable and biodegradable polysaccharide extracted from seaweed. It is composed of two monomers, $\beta$-D-mannuronate and $\alpha$-L-guluronate, known to form gels in the presence of bivalent cations such as calcium $\left(\mathrm{Ca}^{2+}\right)[6,8,9]$. Given these characteristics, this polymer has been widely used for drug and growth factor delivery, cell encapsulation, and as a scaffold in tissue engineering applications $[8,9]$. However, alginate exhibits some limitations in the regard of prospective bone tissue applications, such as the absence of sites for cell attachment or specific receptor interactions, which limits its long-term functionality, further impaired by the swelling-disintegration-erosion of these hydrogels' upon implantation, week biomechanical properties, poor bioresorbability and bioactivity [10-13]. Therefore, the use of nanohydroxyapatite (nanoHA) as a reinforcing component with osteocondutive capabilities can contribute to overcome some of these potential limitations $[4,14]$. NanoHA is commonly used in bone tissue regeneration due to its chemical similarity to the inorganic component of the bone matrix and its inherent characteristics such as biocompatibility, osteoinduction, osteocondutive and osteointegration [4,15,16]. Although in some studies nanoHA was already used to reinforce Alg matrixes [14,17-20], no studies have yet addressed the systematic influence of nanoHA on alginate-based hydrogel systems, neither the effect of distinct nanoHA amounts on its physic-chemical properties and biological response. This is of the utmost relevance, since the amount of nanoHA within the composition of polymer-based hydrogels is crucial to design an efficient composite for bone tissue regeneration. Once nanoHA is gradually degraded, it releases calcium $\left(\mathrm{Ca}^{2+}\right)$ and phosphate $\left(\mathrm{PO}_{4}{ }^{-3}\right)$ ions, which can modulate cellular behavior within the microenvironment, through different signaling pathways, influencing the bone mineralization process and bonding to the surrounding tissues [21]. Accordingly, some authors have reported that the level of solubility, bonelike apatite layer formation, cell attachment, proliferation and differentiation and, ultimately, bone growth rate, could be dependent on the concentration of nanoHA incorporated into polymer-based composites [21-23].

Therefore, an optimizing the ratio composite components must be guaranteed for enhancing its potential clinical application. In this context, the influence of nanoHA concentration on alginate-based hydrogel system was evaluated in terms of: (i) physic-chemical properties (i.e., morphology, chemical analysis, water content, swelling behavior, influence of $\mathrm{pH}$ on degradation and stability, surface charge, release of calcium and phosphate ions, bioactivity response in simulated body fluid), and (ii) biological response, with in vitro cytocompatibility evaluation with human osteoblastic cells and ex vivo functional assessment of bone formation studies, in order to define the best possible combination of Alg-nanoHA for enhancing bone tissue regeneration.

\section{Materials and methods}

\subsection{Preparation of Alg-nanoHA hydrogels}

Initially, a $2 \%(\mathrm{w} / \mathrm{v})$ alginate solution (Alg), was prepared by dissolving the sodium alginate (Sigma-Aldrich) powder in distilled water, at room temperature. Then, this solution was mixed with nanoHA powder (spherical microaggregates of nanoHA with average particle size of $5 \mu \mathrm{m}$, nanoXIM.HAp202, FLUIDINOVA, S.A), at 0, 30, 50 and $70 \mathrm{wt} \%$, during $1 \mathrm{~h}$ at $60 \mathrm{rpm}$. After homogeneity, each mixture was dropped into a $250 \mathrm{mM}$ calcium chloride $\left(\mathrm{CaCl}_{2}\right.$, Sigma-Aldrich) solution, and allowed to harden for $1 \mathrm{~h}$. Spherical hydrogels were collected and washed three times with sterile distilled water. Four hydrogel compositions were produced: Alg (control), Alg-nanoHA30, AlgnanoHA50, Alg-nanoHA70, containing respectively $0,30,50$ and $70 \mathrm{wt}$ $\%$ nanoHA.

\subsection{Characterization of Alg-nanoHA hydrogels}

\subsubsection{Morphology}

The composites matrix was characterized by transmission electron microscopy (TEM, JEOL JEM 1400 TEM). Samples were initially fixed in 2,5\% glutaraldehyde and $2 \%$ paraformaldehyde in cacodylate buffer $0.1 \mathrm{M}$ (pH 7.4) (both Sigma-Aldrich), dehydrated and embedded in Epon resin (TAAB). Ultrathin sections (40-60 nm thick) were stained with uranyl acetate and lead citrate and examined using a JEOL JEM 1400 microscope (TEM). Images were digitally recorded using a CCD digital camera Orious $1100 \mathrm{~W}$. Three samples were used for image acquisition.

External and internal structure and morphology of hydrogels were observed by scanning electron microscopy (SEM). Samples $(n=3)$ were coated with a gold layer (SPI-Module) in an argon atmosphere, and examined using a FEI Quanta 400FEG/ESEM microscope.

\subsubsection{Chemical analysis}

Chemical characterization was performed using attenuated total reflectance - Fourier transformed infrared spectroscopy (ATR - FTIR), with a Perkin -Elmer 2000 FTIR spectrometer. The samples $(n=3)$ were analyzed at a spectral resolution of $2 \mathrm{~cm}^{-1}$ and 100 scans were accumulated per sample.

\subsubsection{Water content and swelling behavior}

Gravimetric method was employed to calculate the water content and swelling ratio, as previously described [24]. Briefly, for water content (W) evaluation, the samples were dried at $60^{\circ} \mathrm{C}$ until a constant weight was reached. The water content was calculated using Eq. (1):

Water content $(\%)=\frac{W_{w}-W_{d}}{W_{w}} \times 100$

where $W_{w}(\mathrm{~g})$ and $W_{d}(\mathrm{~g})$ are the wet and dried weights of the samples, respectively. The results were taken as the mean values of eight measurements.

The swelling behavior was evaluated by measuring the changes in sample weight versus sample immersion time, in phosphate buffer saline (PBS, Sigma-Aldrich). Swelling behavior was evaluated for 28 days at $37^{\circ} \mathrm{C}$, and the media was replaced every 4 days to maintain the initial media volume. The equilibrium swelling ratio was calculated using Eq. (2):

Swelling ratio $(\%)=\frac{W_{f}-W_{i}}{W_{i}} \times 100$

where, $W_{i}$ and $W_{\mathrm{f}}$ are the initial and final weights of the samples, respectively. The results were taken as the mean values of eight measurements.

\subsubsection{Influence of $\mathrm{pH}$ on stability}

The stability of composites, at different $\mathrm{pH}$ values, were evaluated through changes in samples weight, as previously described [6]. The samples were immersed at different $\mathrm{pH}$ values, ranging between 1 and 13 , at $4{ }^{\circ} \mathrm{C}$ for $24 \mathrm{~h}$. Afterwards, they were weighted and the weight alteration ratio (\%), at different $\mathrm{pH}$ was quantified using Eq. (2). The results were taken as the mean values of eight measurements.

\subsubsection{Zeta potential - charge surface}

Zeta potential was determined from streaming potential measurements with a commercial electrokinetic analyzer (EKA, Anton Paar) 
using a special powder cell adapter inside a cylindrical cell. The samples were mounted inside the power cell occupying a volume of about $48.75 \mathrm{~mm}^{3}$, thus maintaining an overall constant height of sample for all measurements. Streaming potential was measured using $\mathrm{Ag} / \mathrm{AgCl}$ electrodes installed at both ends of the streaming channel. The electrolyte used was $1 \mathrm{mM} \mathrm{KCl}$ at $\mathrm{pH}$ of $6.06 \pm 0.10$. Experiments were performed at $24^{\circ} \mathrm{C}$. The conductivity of the electrolyte solution was measured during the assay. The streaming potential was measured while applying an electrolyte flow in alternating directions and pressure ramps from 0 to 200 mbar. For each test, six pressure ramps were performed (three in each flow direction to cope with the asymmetric potential fluctuations). The results were taken as the mean values of eight measurements.

\subsubsection{Release of calcium ions}

The concentration of calcium $\left(\mathrm{Ca}^{2+}\right)$ ions released from composites were determined according to ISO 10993-14 - "Biological evaluation of medical devices- Part 14: Identification and quantification of degradation products from ceramics". Briefly, the samples were placed in Tris [hydroxymethyl] aminomethane- $\mathrm{HCl}$ (Tris-HCl) solution (pH 7.4, Sigma-Aldrich) at $37^{\circ} \mathrm{C}$ and $120 \mathrm{rpm}$. At each time-point, supernatant was collected and filtered $\left(0.2 \mu \mathrm{m}\right.$, Merck). $\mathrm{Ca}^{2+}$ concentration was determined by inductively coupled-plasma atomic emission spectroscopy (ICP-AES). The results were taken as the mean values of five measurements.

\subsubsection{Bioactivity assay in simulated body fluid}

The apatite deposition on composites was evaluated according to Kokubo's method [25]. Samples were immersed in Simulated Body Fluid (SBF) and incubated at $37^{\circ} \mathrm{C}$ and $120 \mathrm{rpm}$, for 7 days. The apatite formation was evaluated by SEM, as described above. The surface elemental composition was carried out by Energy-dispersive X-ray spectroscopy (EDS), and the mass fractions of the elements were quasiquantitatively calculated from at least three large field analysis measurements. Three samples were used for image acquisition and SEMEDS analysis.

\subsection{In vitro cytocompatibility assessment with human osteoblastic cells}

Human mesenchymal stromal cells (hMSCs) isolated from femoral bone marrow, were obtained from an orthopedic surgical procedure, in accordance with established protocols [26]. hMSCs were maintained in minimum essential medium alpha modification ( $\alpha$-MEM), containing $10 \%$ fetal bovine serum (FBS), 100 unit's/mL penicillin, $100 \mu \mathrm{g} / \mathrm{mL}$ streptomycin and $2.5 \mu \mathrm{g} / \mathrm{mL}$ of amphotericin B (all reagents from Gibco). To evaluate the cell response to composites, third subculture cells were seeded at $10^{4}$ cells $/ \mathrm{cm}^{2}$, at $37^{\circ} \mathrm{C}$ for $24 \mathrm{~h}$ in a humidified atmosphere of $5 \% \mathrm{CO}_{2}$. Afterwards, the medium was discarded and the composites were incubated with adherent cells. Cultures, grown in the presence of composites were further characterized throughout the culture time up to 21 days, regarding cell proliferation, metabolic activity, cytoskeletal and mitochondrial organization, cell morphology, alkaline phosphatase activity and gene expression analysis of relevant osteogenic markers. hMSCs grown directly on tissue culture plates, in the absence of composites, were used as controls. The results were taken as the mean values of five measurements.

Additionally, for the analysis of gene expression, an osteogenic-induced control, established in osteogenic-induced conditions - culture medium further supplemented with $10 \mathrm{mM} \beta$-glycerophosphate $10^{-8} \mathrm{M}$ dexamethasone and $50 \mu \mathrm{g} / \mathrm{mL}$ ascorbic acid (all reagents from SigmaAldrich), was established.

\subsubsection{Metabolic activity of the cultures and cell proliferation}

Cultures metabolic activity was evaluated using the MTT assay. At each time-point, cultures were incubated with $10 \%$ MTT solution (3(4,5-dimethyl-2-thiazolyl)-2,5-diphenyl-2H-tetrazolium bromide,
$5 \mathrm{mg} / \mathrm{mL}$, Sigma-Aldrich) for $3 \mathrm{~h}$ at $37^{\circ} \mathrm{C}$. After the incubation period, the culture medium was removed, and the formazan salts were dissolved with dimethylsulfoxide (DMSO, Panreac). Absorbance was determined at $550 \mathrm{~nm}$ on a microplate reader (BioTek).

DNA content was measured using the Quant-iT Picogreen DNA assay (Invitrogen) according to the manufacturer's instructions. Initially, cells were washed with PBS and solubilized with $0.1 \%(\mathrm{v} / \mathrm{v})$ Triton X-100 solution. Cell lysate was then mixed with the Pico-Green solution and incubated in the dark at room temperature for $5 \mathrm{~min}$. The fluorescence intensity was measured with a microplate reader at 485 and $528 \mathrm{~nm}$ for emission and excitation, respectively. The results are expressed in nanograms of DNA.

\subsubsection{Immunofluorescence analysis}

Cell cytoskeleton filamentous actin (F-actin) organization and mitochondrial distribution, as well as the morphology of the cells were assessed by immunofluorescence imaging. Briefly, live cells were incubated with MitoSpy ${ }^{\mathrm{TM}}$ Red CMXRos (250 nM, Biolegend) for $30 \mathrm{~min}$, at $37^{\circ} \mathrm{C}$. Cells were then washed with PBS and fixed with $3.7 \%$ paraformaldehyde for $15 \mathrm{~min}$. Following cells' permeabilization, nonspecific binding sites were blocked with $1 \%$ bovine serum albumin (SigmaAldrich) for $30 \mathrm{~min}$. F-actin was stained with Alexa-Fluor 488 phalloidin-conjugated antibody (1:50, $30 \mathrm{~min}$, Molecular Probes), and nucleus counterstaining with DAPI $(1 \mu \mathrm{g} / \mathrm{mL}, 10 \mathrm{~min}$, Sigma-Aldrich). Images of fluorescent-labelled cells were obtained with a Selena S digital imaging system (Logos Biosystems). Three samples were used for image acquisition.

\subsubsection{Gene expression analysis}

At 21 days of culture, quantitative reverse-transcriptase polymerase chain reaction (qRT-PCR) analysis was performed to evaluate the expression of relevant osteogenesis-related markers. Briefly, total RNA was extracted using TRIZOL ${ }^{\circledast}$ reagent (Invitrogen) and reverse transcribed into complementary DNA (cDNA) with iScript ${ }^{\mathrm{TM}}$ Adv cDNA Kit (BioRad), according to the manufacturer's instructions. The expression of genes: Runt-related transcription factor 2 (RUNX-2, BioRad ID: qHsaCED0044067), Sp7 transcription factor (SP7, BioRad ID: qHsaCED0003759), collagen type I (COL1A1, BioRad ID: qHsaCED0043248) and bone gamma-carboxyglutamate protein (BGLAP, BioRad ID: qHsaCED0038437), was quantitatively determined in a RT-PCR equipment (CFX96, BioRad) using $\mathrm{iQ}^{\mathrm{TM}} \mathrm{SYBR}^{\oplus}$ Green Supermix (BioRad). Beta-actin (ACTB, qHsaCED0036269) gene was used as the reference gene for normalization. The cycling conditions were as follows: an activation cycle at $95^{\circ} \mathrm{C}$ for $3 \mathrm{~min}$, followed by 40 cycles of $95^{\circ} \mathrm{C}$ degeneration for $10 \mathrm{~s}, 60^{\circ} \mathrm{C}$ annealing and extension for $30 \mathrm{~s}$. The melting curve analysis was carried out on each sample to ensure a single amplicon at $55-95^{\circ} \mathrm{C}$ for $10 \mathrm{~s} /$ cycle, with $0.5^{\circ} \mathrm{C}$ increment each cycle. The cycle threshold $(\mathrm{Ct})$ for each transcript expression was export from Bio-rad CFX maestro software. The relative intensity of each target gene was normalized to ACTB levels, and calculated via the $2^{-\Delta \Delta \mathrm{Ct}}$ method.

\subsection{Ex vivo functional assessment of bone formation}

The biofunctionality of composites was assayed ex vivo in an embryonic chick femoral segmental defect model. Briefly, femurs were dissected from day 11 chick embryos (Gallus domesticus), where the soft tissues, such as adherent muscles and ligaments, were carefully removed while preserving the periosteum. The femurs were cut at mid diaphysis for the establishment of a segmental defect, with a number 11 scalpel blade. Femurs, with composites being implanted within the middiaphysis defect, were carefully settled onto Netwell ${ }^{\mathrm{TM}}$ Insert $(440 \mu \mathrm{m}$ mesh size polyester membrane, Corning) in 6 well plates. Implanted femurs were maintained for 11 days in minimum essential medium ( $\alpha$ MEM), containing ascorbic acid $(50 \mu \mathrm{g} / \mathrm{mL})$, penicillin (100 units $/ \mathrm{mL}) /$ streptomycin $(100 \mu \mathrm{g} / \mathrm{mL})$ and $2.5 \mu \mathrm{g} / \mathrm{mL}$ amphotericin $\mathrm{B}$, at liquid/gas 
interface, in a humidified atmosphere of $5 \% \mathrm{CO}_{2}$ and $37^{\circ} \mathrm{C}$. Culture media was changed daily for the duration of the experiment. At the end of the culture period, femurs were fixed in $4 \%$ paraformaldehyde (Sigma-Aldrich) and prepared for histological examination. Samples were dehydrated through a series of graded alcohols, cleared in Histoclear $^{\circledast}$ and embedded in paraffin. Tissue sections $6 \mu \mathrm{m}$ thick were cut from across the femur samples and stained with Alcian blue/Sirius red (AB/SR), Masson's trichrome or von Kossa staining, as previously described [27]. Images were captured with an Olympus BX-51/22 dotSlide digital virtual microscope and created using OlyVIA 2.1 software (Olympus Soft Imaging Solutions, $\mathrm{GmBH})$. Eight femurs $(n=8)$ per experimental condition, with a total of six sections from each femur for each histological stain were evaluated.

\subsection{Statistical analysis}

All experiments were performed in triplicate as independent experiments. The results were reported as the arithmetic mean \pm standard deviation. The experimental data were analyzed using IBM $^{\circledR}$ SPSS ${ }^{\circledR}$ Statistics (vs. 22.0, Statistical Package for the Social Sciences Inc). The one-way analysis of variance (ANOVA) followed by the post hoc Turkey HSD multiple comparison tests were used to determine the significant difference $(\mathrm{p}<0.05)$.

\section{Results}

\subsection{Alg-nanoHA hydrogel system characterization}

\subsubsection{Morphology characterization}

Fig. 1 presents digital images of composites that were obtained by mixing Alg and nanoHA, at different weight percentages (0, 30, 50 and $70 \mathrm{wt} \%$ ), into spherical hydrogels, via external gelation. Overall, the samples showed tightly knit and smooth surface, and a whitened coloration proportional to the amount of nanoHA added (Fig. 1A), which also led to a slight increase in terms of area and weight, although no significant differences were found between different conditions (Table 1).

The matrix, structure and morphology of composites is shown in Fig. 1B and C. TEM analysis allowed to characterize the Alg matrix as network of fibril-like structures, and to observe that nanoHA content increased the crosslinking of the Alg network (Fig. 1B). Through SEM analysis, it was shown that all samples present a lamellar structure with irregular pores in the inner layer (Fig. 1C). Alg hydrogels showed a smooth surface, whereas Alg-nanoHA showed a rougher surface, as comparing to control, due to the presence of nanoHA (Fig. 1C). The nanoHA particles were well embedded and homogeneously dispersed throughout the Alg matrix, both externally and internally (Fig. 1C).

\subsubsection{Chemical analysis}

The ATR - FTIR spectra (Fig. 2A) shows the chemical composition of the hydrogels. Alg displayed the characteristic alginate bands, as the asymmetric and symmetric stretching of carboxylic group $\left(\mathrm{COO}^{-}\right)$on the polymeric backbone, found at 1592 and $1417 \mathrm{~cm}^{-1}$, respectively. The broad peak spread over the range 3311 to $3307 \mathrm{~cm}^{-1}$ corresponds to hydroxyl group $\left(\mathrm{OH}^{-}\right)$. The bending of the $\mathrm{OH}^{-}$group of the carboxyl is depicted at $819 \mathrm{~cm}^{-1}$. ATR - FTIR spectra of the Alg-nanoHA showed a shift of the $\mathrm{COO}^{-}$bands towards lower wavelengths, proportionally to the increase in nanoHA ratio. Bending and stretching of the phosphate group $\left(\mathrm{PO}_{4}{ }^{3-}\right)$ were observed at $1020 \mathrm{~cm}^{-1}$ and $559 \mathrm{~cm}^{-1}$, respectively, that are attributed to the overlap of $\mathrm{COO}^{-}$stretching of $\mathrm{Alg}$ and $\mathrm{PO}_{4}{ }^{3-}$ stretching of nanoHA. Moreover, their intensity increased with increasing of nanoHA content. The presence of bands at $3365 \mathrm{~cm}^{-1}$ and $629 \mathrm{~cm}^{-1}$ was assigned to $\mathrm{OH}^{-}$group, corresponding to lattice water.

\subsubsection{Water content and swelling behavior}

Table 1 shows the water content and swelling ratio of the composites. The water content was inversely proportional to the amount of nanoHA (Table 1). No significant differences in the water content were observed between Alg (control) and, Alg-nanoHA30, and Alg-nanoHA50 hydrogels. However, the water content of Alg-nanoHA70 was significantly lower compared to Alg (control) and other Alg-nanoHA hydrogels (Table 1).

The swelling behavior of the hydrogels was evaluated by measuring the changes in sample weight over time. No weight differences were observed between samples over time (data not shown). For all samples, the swelling equilibrium was observed after $18 \mathrm{~h}$. The swelling ratio was inversely proportional to the nanoHA amount (Table 1). All AlgnanoHA hydrogels showed significantly lower swelling ratio compared to Alg (control). However, no significant differences on the swelling ratio were observed between Alg-nanoHA30 and Alg-nanoHA50. While, the swelling ratio of Alg-nanoHA70 was significantly lower than that of Alg-nanoHA30 and Alg-nanoHA50.

\subsubsection{Zeta potential - charge surface}

Zeta potential values of composites are presented in Table 1. All samples showed a negative zeta potential, which was affected by high nanoHA content. No differences of zeta potential values were found between Alg and Alg-nanoHA30. However, Alg-nanoHA50 and AlgnanoHA70 showed higher zeta potential values than that of Alg (control) and Alg-nanoHA30 hydrogels.

\subsubsection{Influence of $\mathrm{pH}$ on stability}

In Fig. 2B the stability of the composites at different $\mathrm{pH}$ values are presented. The introduction of nanoHA did not affect the behavior of hydrogels, in which a shrinkage and breakdown of the matrixes were verified, at acid and alkaline $\mathrm{pH}$ values, respectively. However, composites stability was proportional to the nanoHA content. The Alg-nanoHA hydrogels were more stable at higher $\mathrm{pH}$ values compared with Alg (control).

\subsubsection{Release of calcium ions}

Fig. 2C depicts the released $\mathrm{Ca}^{2+}$ concentration from hydrogels detected by ICP-AES.

At day 1 , the $\mathrm{Ca}^{2+}$ release from $\mathrm{Alg}$ (control) was $86 \mathrm{ppm}$, while from Alg-nanoHA hydrogels, the $\mathrm{Ca}^{2+}$ concentration was proportional to the amount of nanoHA. The released $\mathrm{Ca}^{2+}$ concentration maintained constant for all composites until day 3. Afterward, this period, an increase of released $\mathrm{Ca}^{2+}$ concentration released was observed for AlgnanoHA hydrogels, where, from Alg-nanoHA30, the $\mathrm{Ca}^{2+}$ ions release was significantly lower than released from Alg-nanoHA50 and AlgnanoHA70 throughout the 28 days of incubation.

\subsubsection{Bioactivity assay in simulated body fluid}

The bioactivity of developed composites was addressed by SEM imaging and EDS spectra, after 7 days' immersion in SBF solution (Fig. 3). No apatite crystals deposition was observed on Alg hydrogel, while on Alg-nanoHA, an increase of apatite crystals deposition was observed, evidencing bumps and clusters of apatite's crystals on the composites' surface. Through EDS elemental analysis, the chemical composition of these crystals were identified, attesting to be CaP particles. On Alg surface, only Ca was detected, whereas, on Alg-nanoHA surfaces $\mathrm{Ca}$ and $\mathrm{P}$ were identified, confirming its surface apatite mineralization (Fig. 3). EDS semi-quantitative analysis revealed an increase of $\mathrm{Ca} / \mathrm{P}$ ratio proportional to the content of nanoHA within the composite: Alg-nanoHA30 - $\mathrm{Ca} / \mathrm{P}=1.04$, Alg-nanoHA50 - $\mathrm{Ca}$ / $\mathrm{P}=1.44$, and Alg-nanoHA70 $-\mathrm{Ca} / \mathrm{P}=1.60$.

\subsection{In vitro cytocompatibility assessment with human osteoblastic cells}

\subsubsection{Metabolic activity of the cultures and cell}

The hMSCs cultures metabolic activity and cell proliferation were evaluated by MTT assay (Fig. 4A) and DNA quantification (Fig. 4B), 

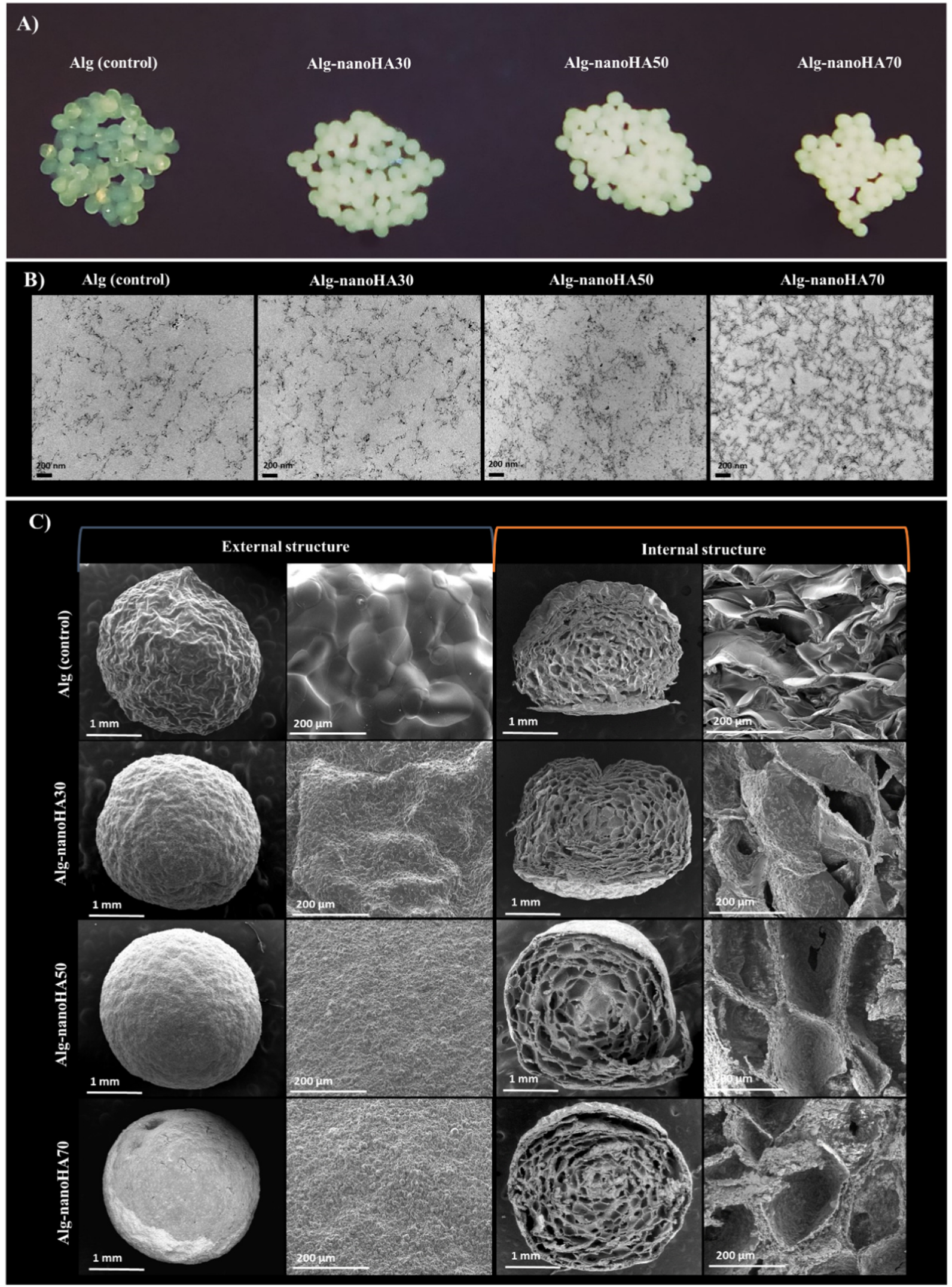

Fig. 1. (A) Digital images of composites with different nanoHA weight ratios (0, 30, 50 and 70\%). (B) Matrix of hydrogels observed by TEM. Scale bar corresponds to $200 \mathrm{~nm}$. (C) SEM micrographs of external and internal structure of composites. Scale bars correspond to $1 \mathrm{~mm}$ and $200 \mu \mathrm{m}$. 
Table 1

Physical characteristics of the composites.

\begin{tabular}{|c|c|c|c|c|c|}
\hline Hydrogels & Weight $(\mathrm{g})$ & Area $\left(\mathrm{cm}^{2}\right)$ & Water content $(\%)$ & Swelling ratio (\%) & Zeta potential $(\mathrm{mV})$ \\
\hline Alg (control) & $0.021 \pm 0.003$ & $0.270 \pm 0.013$ & $95.9 \pm 1.6$ & $172.7 \pm 6.8$ & $-14.358 \pm 1.431$ \\
\hline Alg-nanoHA30 & $0.021 \pm 0.003$ & $0.283 \pm 0.013$ & $94.8 \pm 1.7$ & $150.1 \pm 5.5^{*}$ & $-13.144 \pm 1.670$ \\
\hline Alg-nanoHA50 & $0.022 \pm 0.003$ & $0.291 \pm 0.012$ & $93.1 \pm 1.5$ & $149.6 \pm 4.1^{*}$ & $-9.111 \pm 1.812^{*, \#}$ \\
\hline Alg-nanoHA70 & $0.025 \pm 0.003$ & $0.303 \pm 0.013$ & $88.2 \pm 1.1^{*, \#}$ & $104.3 \pm 6.0^{*, \#}$ & $-9.747 \pm 0.741^{*, \#}$ \\
\hline
\end{tabular}

Data are expressed as mean $\pm \mathrm{SD}(\mathrm{n}=8)$.

* Significant differences compared to Alg (control), with $p<0.05$, obtained by Tukey's post-hoc test;

\# Significant differences different between Alg-nanoHA hydrogels, with $\mathrm{p}<0.05$, obtained by Tukey's post-hoc test.

(a)

(b)
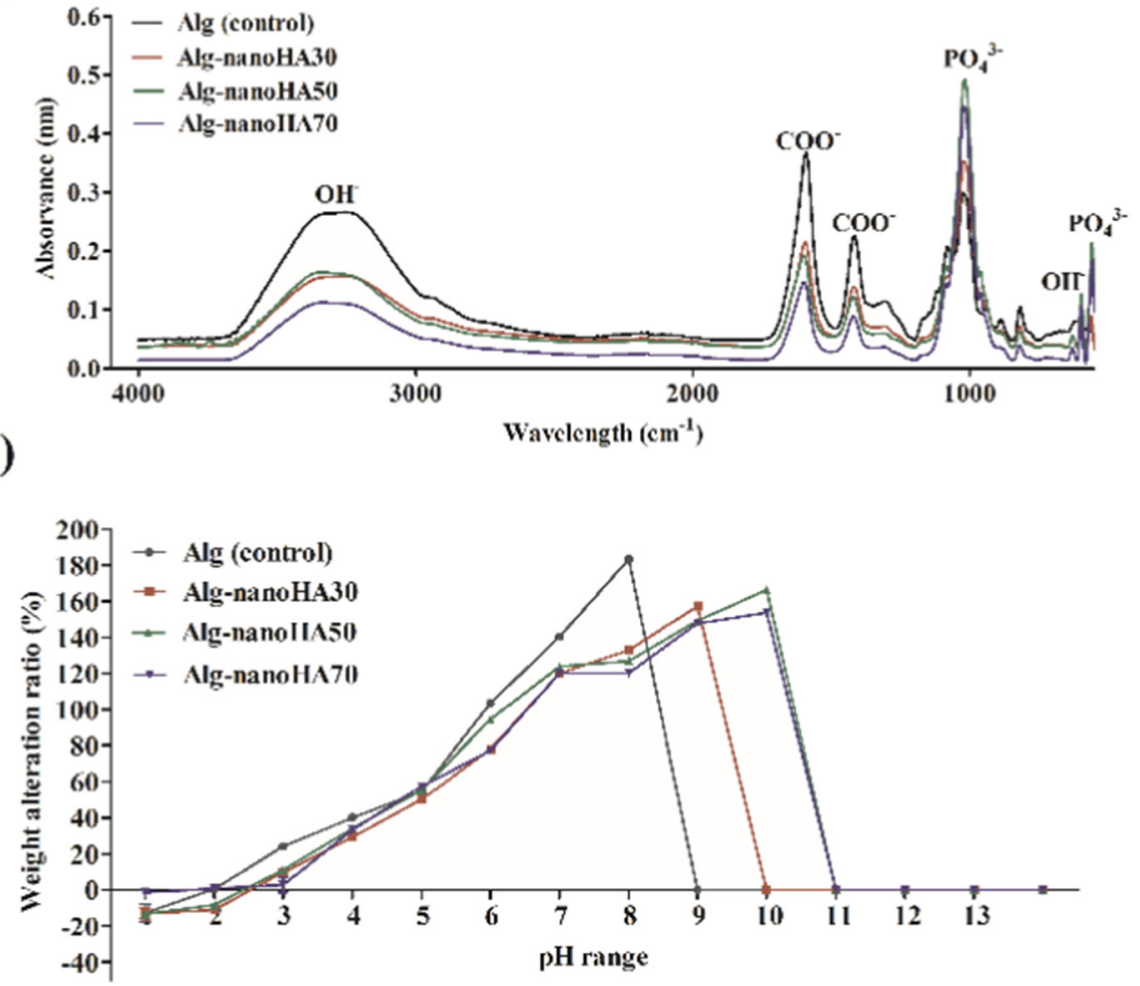

(c)

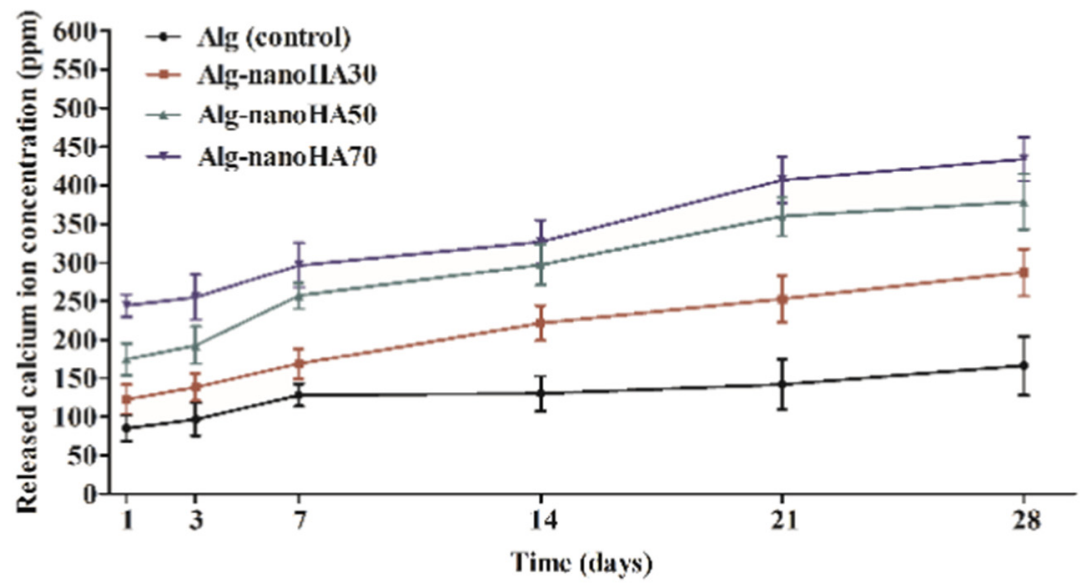

Fig. 2. (A) ATR-FTIR spectra of composites. (B) Stability of the hydrogels at different $\mathrm{pH}$, after $24 \mathrm{~h}$ incubation. (C) Released $\mathrm{Ca}^{2+}$ ions concentration from composites over time. Data are expressed as mean \pm SD (FTIR $(n=3)$; pH stability $(n=8)$ and released calcium $(n=5))$. 

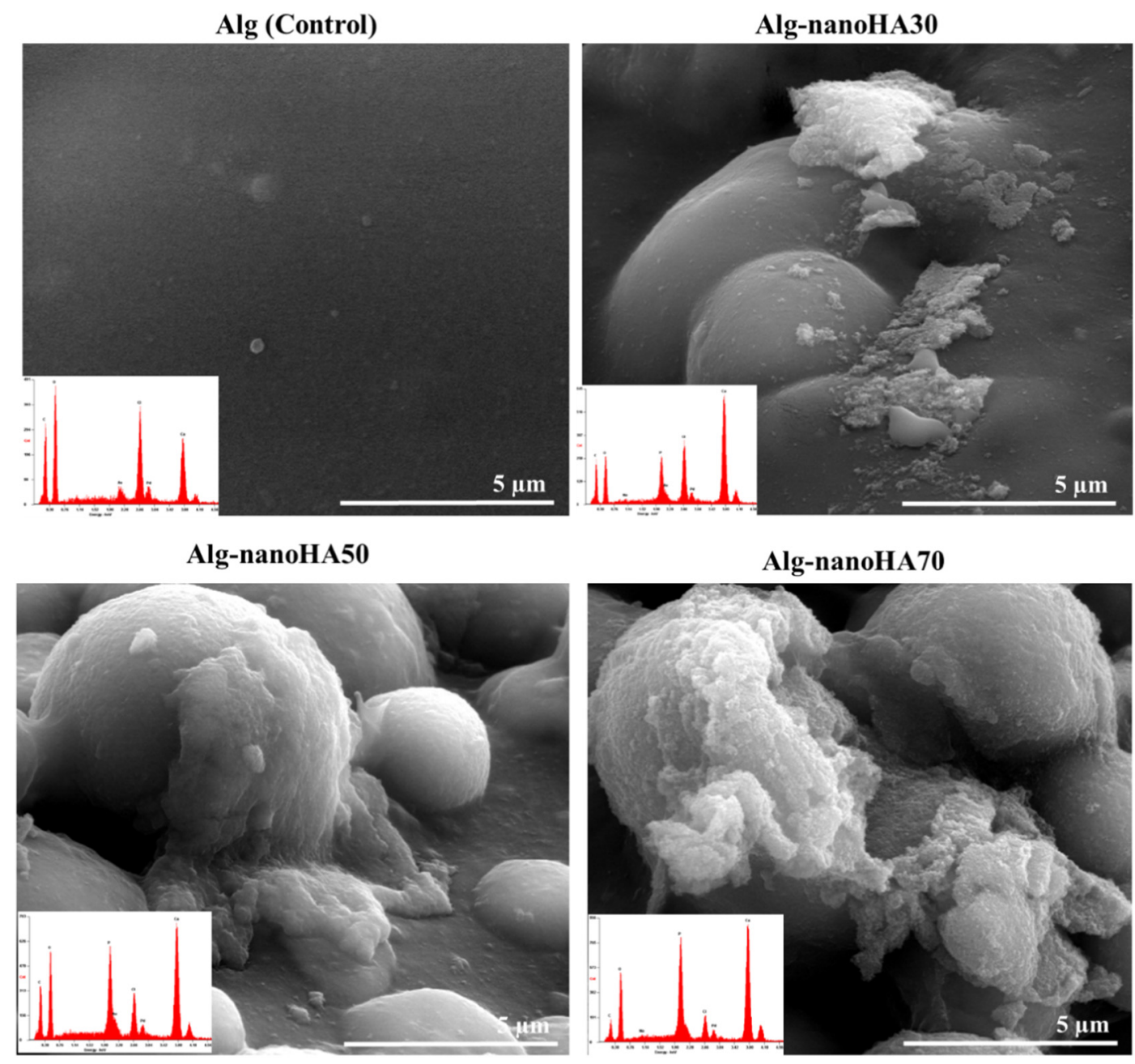

Fig. 3. SEM images and EDS spectra of composites surface after immersion in SBF for 7 days. Scale bar corresponds to $5 \mu$ m.

respectively. Either in the control conditions or in the presence of hydrogels, hMSCs metabolic activity increased throughout the culture period with no significant differences between experimental conditions, at any time of incubation (Fig. 4A). Regarding total DNA content, in control conditions, an increase level was attained throughout the culture time suggesting an active cell proliferation throughout the entire culture period. Comparatively, Alg and Alg-nanoHA30 hydrogels induced a similar cell proliferation whereas Alg-nanoHA50 and Alg-nanoHA70 showed a significantly reduced cell proliferation, at day 14 and 21, as compared to the other experimental conditions (Fig. 4B).

\subsubsection{Immunofluorescence analysis}

The hMSCs cytoskeleton, mitochondrial organization and morphology of cells grown in the presence of developed hydrogels were assessed by immunofluorescence following F-actin and mitochondrial staining, and nucleus counterstaining. Representative micrographs of the cellular culture at day 3 and day 14 are presented on Fig. 5. In control conditions, at day 3, cells presented a dense network of F-actin filaments arranged as stress fibers, with cells presenting a flattened and elongated morphology with evident cytoplasmic extensions and long filopodia. Mitochondrial probe revealed an organized tubular network of fused mitochondria, with a preferential perinuclear localization. In addition, cells were found to establish prominent cell-to-cell contacts. Cultures grown on the presence of Alg or Alg-nanoHA hydrogels presented a similar morphology and mitochondrial structure, inducing a similar cytoplasmic organization, F-actin polymerization and mitochondrial arrangement under the different experimental conditions.
On day 14, established cultures presented a confluent cell layer within control and in cultures grown with Alg or Alg-nanoHA hydrogels. Cells presented an elongated morphology and a dense mitochondrial network with perinuclear organization.

\subsubsection{Gene expression analysis}

Gene expression analysis was carried out by quantitative RT-PCR in order to address the osteogenic activation of hMSCs, grown in the presence of developed composites (Fig. 6). hMSCs (control) were grown under basal and osteogenic inducing conditions and were assessed through the expression of the osteogenic transcription factors Runx2 and Sp7, as well as their downstream effectors Colla1 and BGLAP.

Regarding the expression of Runx2, cultures grown in the presence of Alg presented a similar expression as compared to basal control. Cultures grown in the presence of Alg-nanoHA presented a significantly higher level than basal control and Alg, that, in the case of AlgnanoHA30 and Alg-nanoHA50, were further found to be significantly higher than the osteogenic-induced control. Regarding Sp7, Col1a1 and BGLAP expression, cultures grown in the presence of Alg hydrogels presented significantly higher levels than basal control. Cultures grown in the presence of Alg-nanoHA hydrogels, particularly Alg-nanoHA30 and Alg-nanoHA50, significantly increased Sp7, Col1a1 and BGLAP expression to levels similar to those attained in osteogenic-induced cultures. 
a)

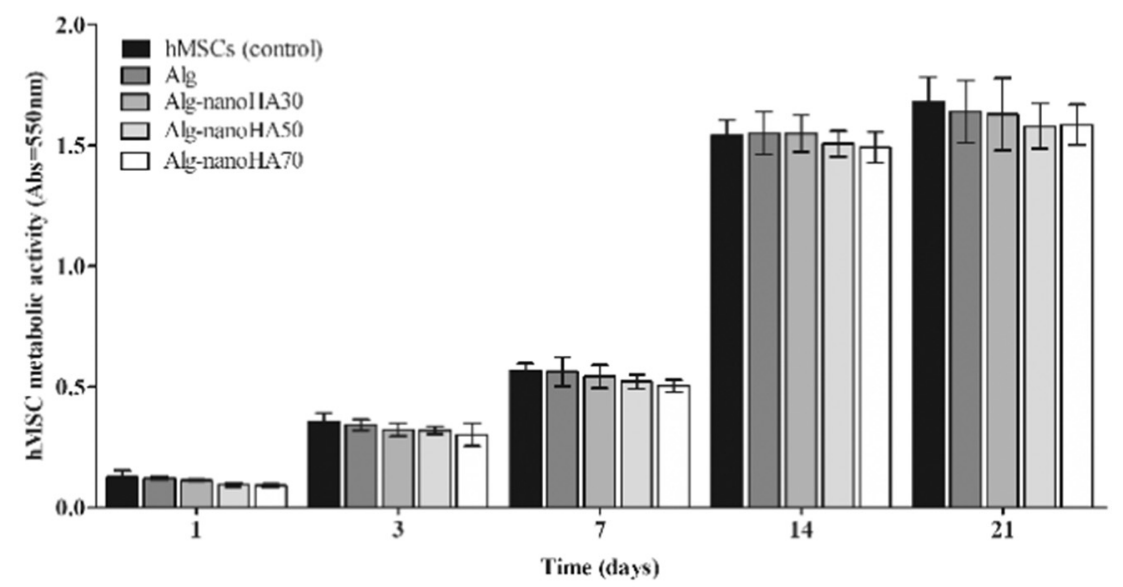

b)

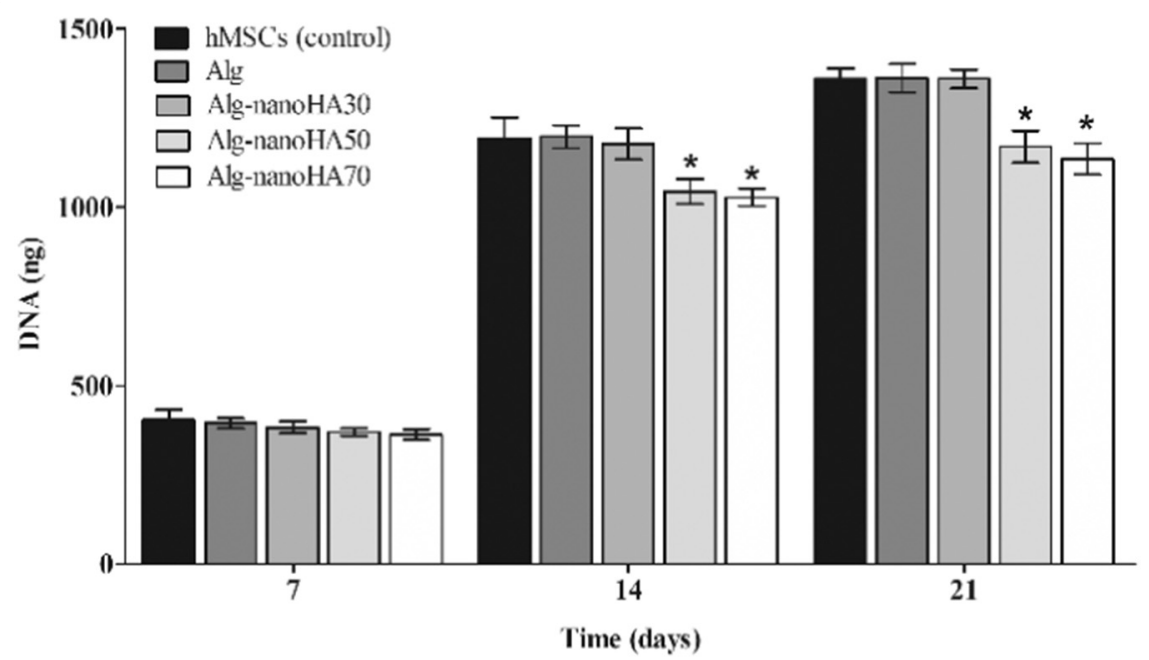

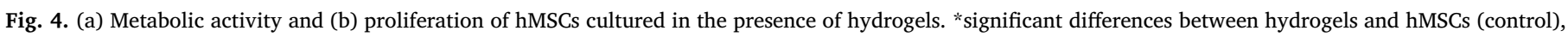
for each time point, with $\mathrm{p}<0.05$, obtained by Tukey's post-hoc test. Data are expressed as mean \pm SD ( $n=5$ ).

\subsection{Ex vivo functional assessment of bone formation}

To address the biofunctionality of developed composites, these were implanted in segmental mid-diaphysis defects in embryonic chick femurs, grown for 11 days. Histological assessment of the implanted femurs is presented on Fig. 7.

Femurs cultured in the absence (control) and presence of Alg hydrogels moderately induced the osteogenic activation, with evidence of collagen deposition at the defect margin, as observed by the red coloration in SR/AB staining and corresponding blue coloration within Masson's trichrome staining. Evidence of tissue mineralization was further attained at the developing bone collar within the peripheral structure of the tissue, as observed by von Kossa staining. Interestingly, Alg-nanoHA hydrogels induced a distinctive biological response. AlgnanoHA30 and Alg-nanoHA50 enhanced the osteogenic response; both induced collagen deposition at the marginal bone collar and within the central region of the bone diaphysis, as sustained by the more intense collagen-positive matrix identified on histochemical staining. In addition, an organized pattern of thick mineralized trabecula was identified within the collar structure of the diaphysis. Comparatively, a significantly higher osteogenic activation was attained with AlgnanoHA30, with a strong collagenous matrix being deposited within the central and marginal regions of the diaphysis, in close association with an organized and thick mineralized trabecular structure. Contrariwise, the implantation of Alg-nanoHA70 induced a reduced collagen deposition at the defect margin, as observed by both histochemical staining techniques. Furthermore, a thinner trabecular organization within the collar bone at the diaphysis was verified, with lessened mineralized trabecular organization.

\section{Discussion}

Composites, combining ceramics and polymers, have emerged as potential biomaterials for the management of bone defects [6]. The osteoconductive/osteoinductive, biocompatibility and bioactivity properties of nanoHA materials $[7,28]$ combined with the injectability, biocompatibility and biodegradation properties of Alg polymers $[29,30]$ can improve the physic-chemical and cell adhesion/tissue development of the attained composites, enhancing bone tissue regeneration [31-33]. However, these properties could be dependent on the amount of ceramic nanoparticles used within the polymer-based composite, as observed by other authors [21-23]. In this context, in the present study, the influence of nanoHA content on an alginate-based hydrogel system was studied, regarding its physic-chemical and biological properties, within in vitro and ex vivo studies, aiming to optimize the combination of Alg and nanoHA within the established system for 

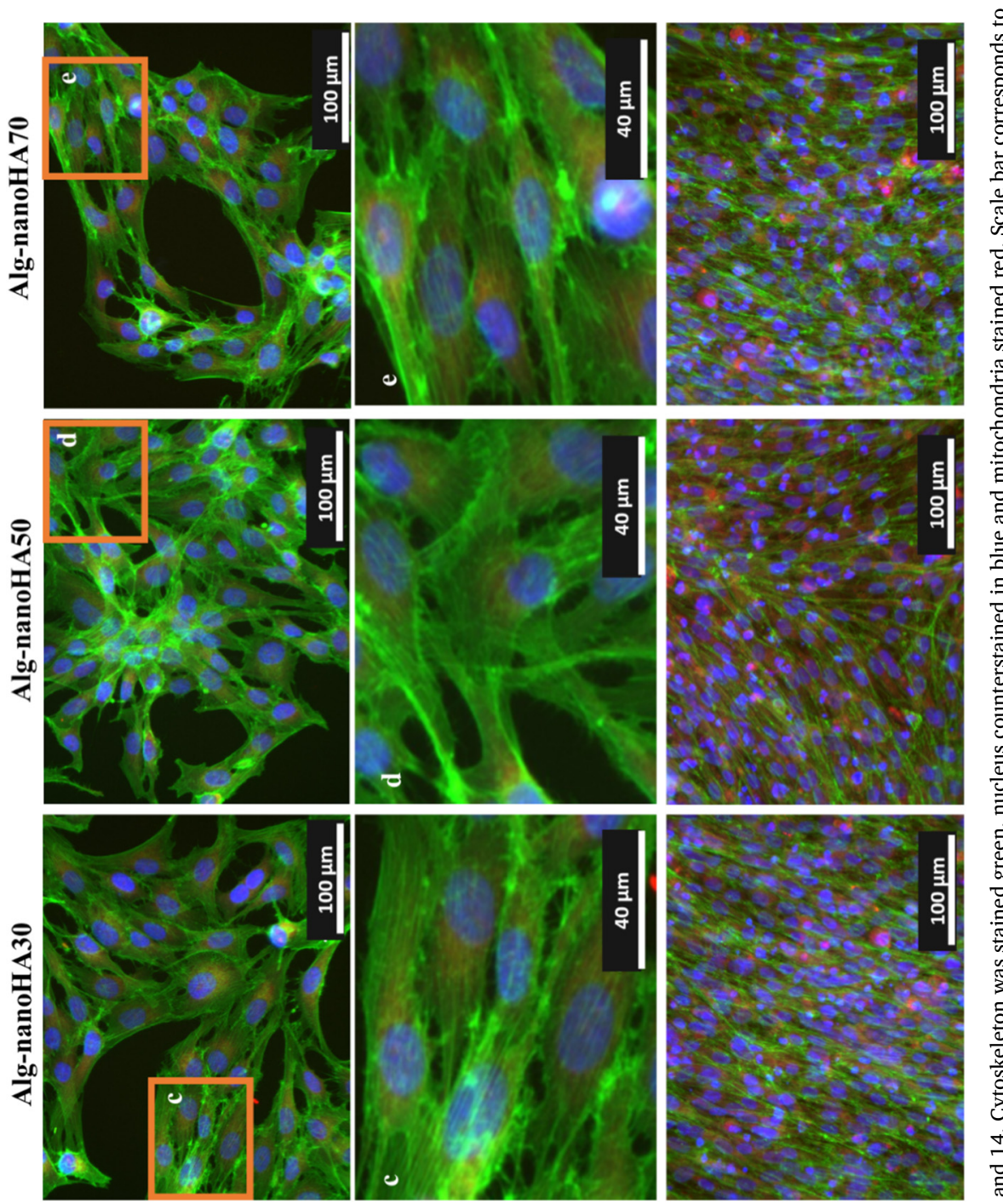

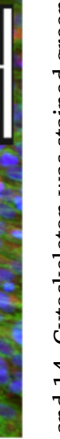
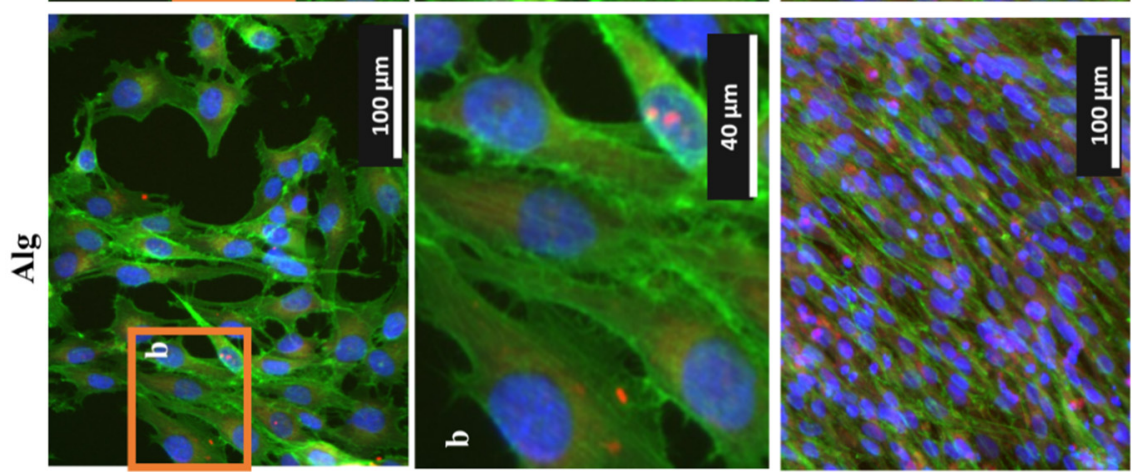

目

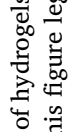
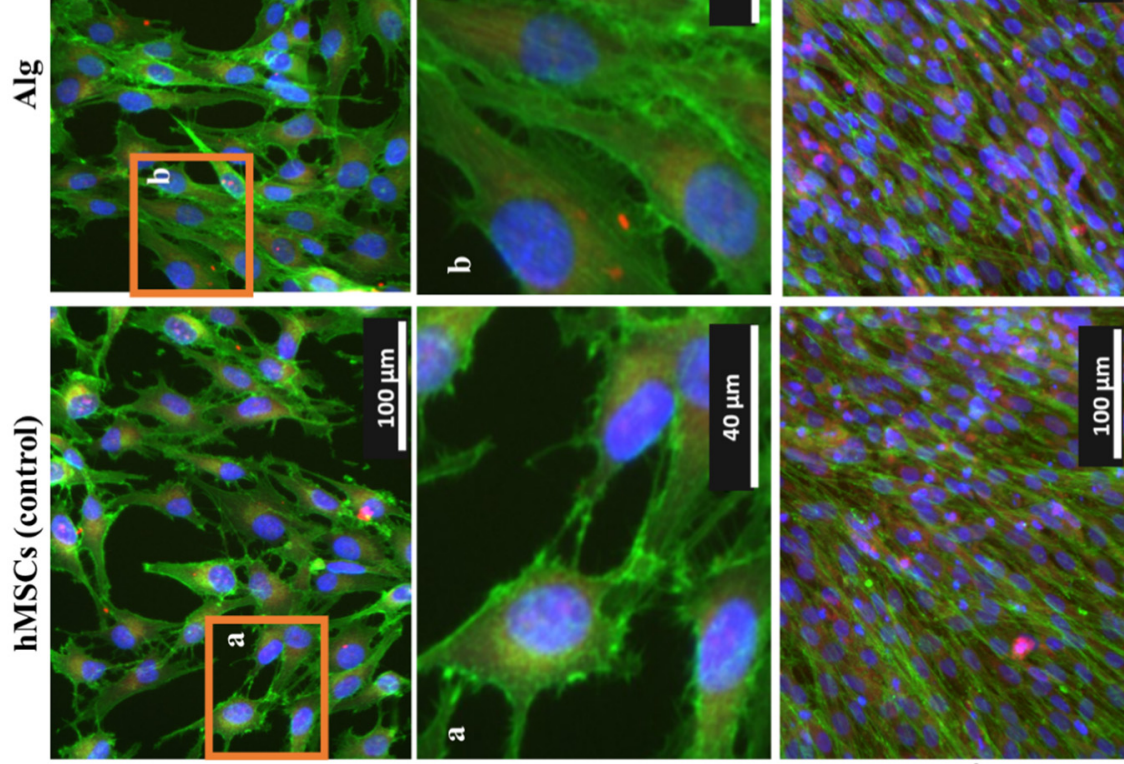

E K

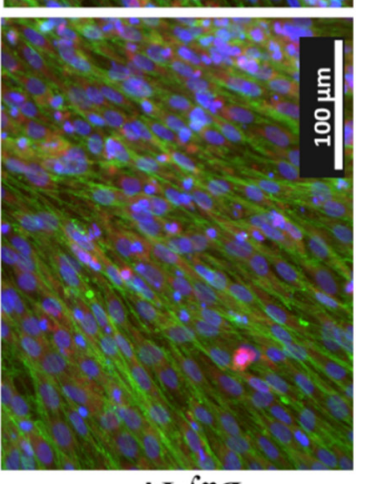

tI KEG

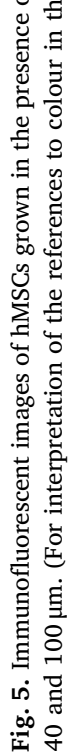




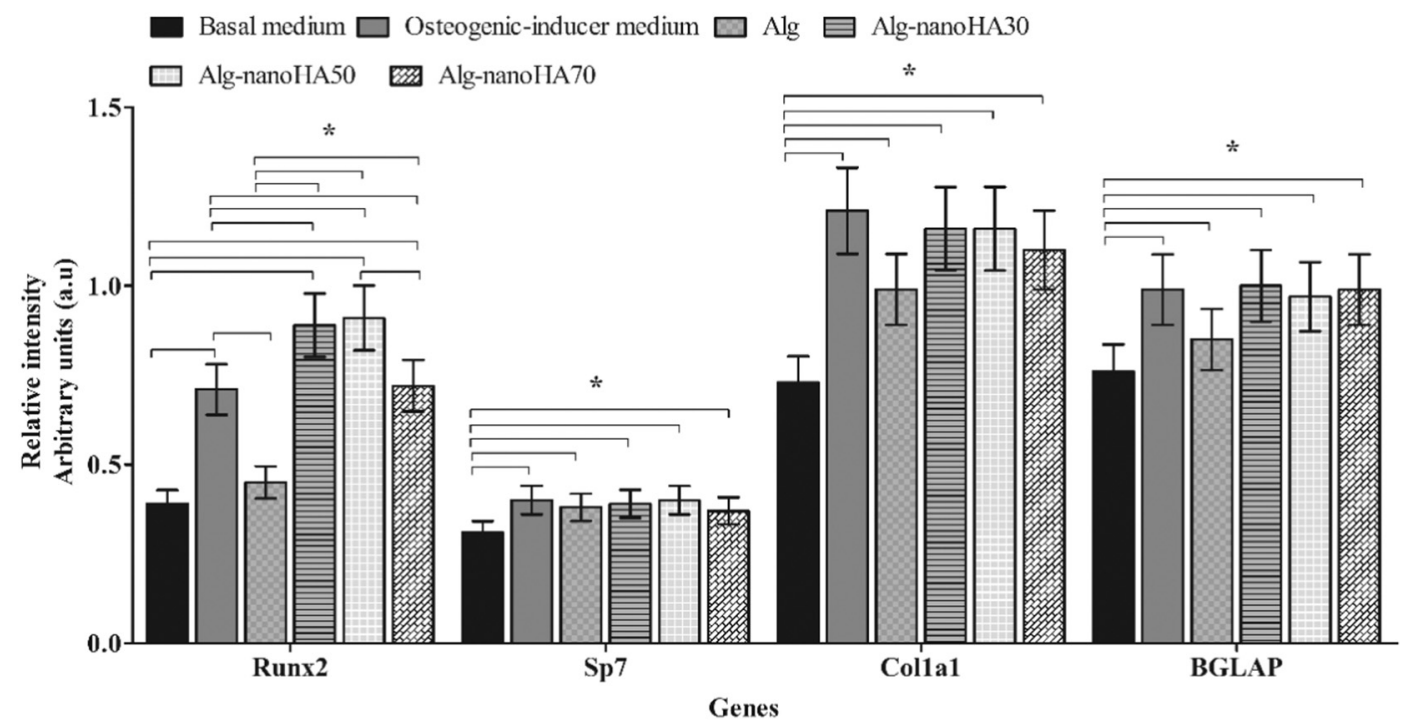

Fig. 6. Gene expression of hMSCs cultured in basal medium, osteogenic-inducer medium, Alg and Alg-nanoHA hydrogels. *Significant differences between all conditions used, with $\mathrm{p}<0.05$, obtained by Tukey's post-hoc test. The relative intensity of each target gene was normalized to beta-actin levels (ACTB), and calculated via the $2^{-\Delta \Delta \mathrm{Ct}}$ method. Data are expressed as mean $\pm \mathrm{SD}(\mathrm{n}=3)$. Related transcription factor 2 (RUNX-2), Sp7 transcription factor (SP7), collagen type I (COL1A1) and bone gamma-carboxyglutamate protein (BGLAP).

maximizing the bone regeneration process.

Initially, physic-chemical characteristics of Alg-nanoHA hydrogels were addressed. Alg-nanoHA hydrogels, regardless of the nanoHA content, showed a considerable matrix strength, stiffness and permeability, typical of this type of gelation [34]. The characteristic absorption bands of Alg and nanoHA can be clearly seen in the ATRFTIR spectra, indicating a successful entrapment of nanoHA into the Alg matrix, without chemical reactions occurring between them. The
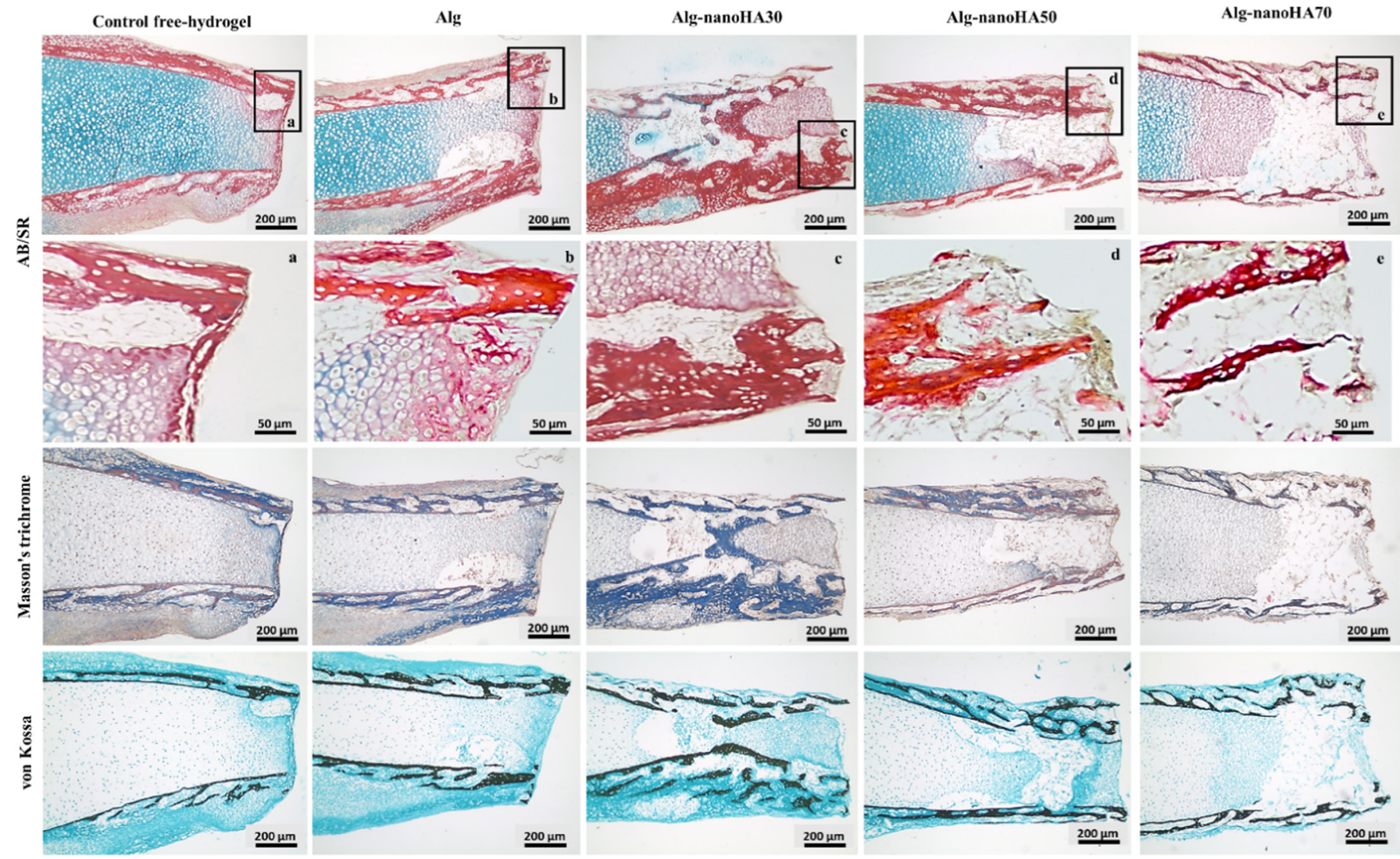

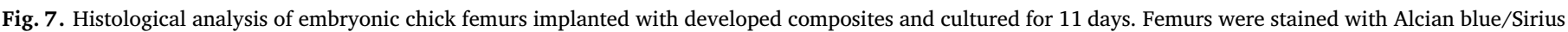

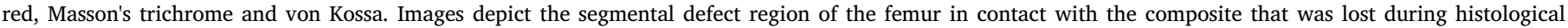

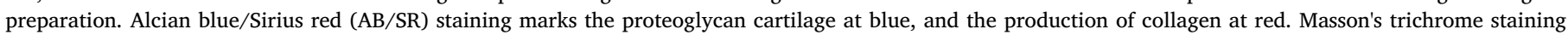

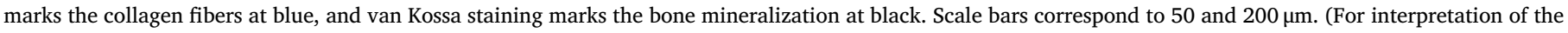
references to colour in this figure legend, the reader is referred to the web version of this article.) 
entrapment of nanoHA increased the crosslinking of the polymeric network, mainly when nanoHA was added at $70 \%$. This phenomenon was probably due to the ability of the $\mathrm{Ca}^{2+}$ ions, from the nanoHA particles, induces a specific and strong interaction with G-blocks of Alg, increasing the structural cohesion and leading to a stronger matrix with low solubility in water [35]. Moreover, nanoHA particles promoted the formation of rougher surfaces. Some authors have reported that rough implant surfaces, e.g. nanophase titania, zinc oxide or bioglass polymer composites, allow enhanced tissue ingrowth and improve the cell adhesion, proliferation and differentiation compared to smooth polymeric substrates [36,37]. All Alg-nanoHA hydrogels showed interconnected pores, thus contributing to the retention of a large amount of water in the alginate matrix and further promoting diffusion of nutrients and macromolecules into the structure, following tissue implantation $[23,38]$.

Water-swelling characteristics of composites play an important role in the absorption of body fluids and transfer of cell nutrients and metabolites $[39,40]$. The interactions of carboxyl $(-\mathrm{COOH})$ and hydroxyl $(-\mathrm{OH})$ groups of the polymeric network with water molecules, leads to their diffusion into the hydrogel driven by the osmotic pressure [17]. However, the water-swelling content of composites decreased with the inclusion of nanoHA, with a significant impact when nanoHA was added at $70 \%$. The presence of nanoHA, particularly at high level, could further contract and restrict the movability of the Alg polymeric chains, creating elastic forces within the hydrogel network, providing resistance to water diffusion and swelling tendencies $[17,39]$ and, consequently, affecting the diffusivity of nutrients, proteins and metabolites and cell-matrix interactions [17].

The balance between the attractive and repulsive forces in composites was determined by zeta potential measurements [9]. Several authors have shown that low zeta potential values in sodium alginate, carragennan, chitosan, and galactomannans might be associated with a higher concentration of $\mathrm{Ca}^{2+}$ ions [9,41,42]. Thereby, with the introduction of nanoHA, there was an increase of $\mathrm{Ca}^{2+}$ ions within the polymeric matrix, which could have contributed to increase the ionic strength of the network, consequently bringing zeta potential closer to the isoelectric point [42], which was particularly evident for Alg-nanoHA50 and Alg-nanoHA70. Some authors have reported that the negative surface charge of sheet-shaped hydrogels and calcium composites can positively affect the protein adsorption, osteoblast attachment and proliferation $[43,44]$.

The $\mathrm{pH}$ of tissue fluids undergoing repairing events may play a regulatory role in the healing and mineralization processes of bone since, at the early healing phase, tissue $\mathrm{pH}$ is lower than normal physiological $\mathrm{pH}$ (7.2), owing to accumulation of acidic metabolites in tissue fluids [45]. Furthermore, during the mineralization phase, an increase in tissue calcium content occurs due to the mineral deposition associated with new bone formation which promotes the increase in $\mathrm{pH}$ to reach more alkaline values [45]. The composites used in the present study showed to be stable at a large range of $\mathrm{pH}$ values, in which their behavior and stability were found to be influenced by the nanoHA content. At acidic conditions occurs the protonation of the $\mathrm{COO}^{-}$groups of the Alg network [46], and the presence of nanoHA increases $\mathrm{Ca}^{2+}$ concentration and consequently the degree of network cross-linking, promoting the shrinkage of the composites. Under alkaline conditions occurs the ionization of $\mathrm{COO}^{-}$groups of $\mathrm{Alg}$ and the diffusion of $\mathrm{Ca}^{2+}$ ions from gelling sites, endorsing the swelling of composites [46]. The high stability of Alg-nanoHA hydrogels at high $\mathrm{pH}$ values may be further related to the stability of nanoHA at neutral and alkaline environments [47].

Additionally, the bioactivity response of the composites was characterized regarding the apatite deposition on the materials' surface, upon immersion in SBF. The formation of bone-like mineral on the surfaces is correlated with an increase in the implant's calcium phosphate solubility [48] and is an essential property to indicate the bonding with living bone $[48,49]$. No mineral layer was observed over
Alg hydrogels, but the presence of nanoHA induced significantly the mineral layer growth with apatite structures developing on Alg-nanoHA hydrogels, which increased with increasing nanoHA content. This increase of apatite on Alg-nanoHA surfaces is probably due to the availability of negatively charged hydroxyl groups on nanoHA that acted as a nucleation site for crystal deposition $[48,49]$. However, the observed $\mathrm{Ca} / \mathrm{P}$ molar ratios were lower than that of stoichiometric hydroxyapatite (HAP), i.e. 1.67 [50]. Several authors have shown that apatite coatings via $\mathrm{SBF}$ incubation can have lower $\mathrm{Ca} / \mathrm{P}$ ratios than stoichiometric HA [50,51]. Once, the apatite's in vertebrate bone and enamel is not pure hydroxyapatite, it contains other ions, including, $\mathrm{CO}_{3}{ }^{2-}, \mathrm{Cl}^{-}$, $\mathrm{Mg}^{2+}, \mathrm{Na}^{+}$, and $\mathrm{K}^{+}$. Small amounts of some of these ions (i.e. $\mathrm{Mg}^{2+}$, $\mathrm{Na}^{+}$) can substitute for $\mathrm{Ca}^{2+}$ ions in the crystal lattice resulting in a lower $\mathrm{Ca} / \mathrm{P}$ ratio $[50]$.

In fact, the presence of nanoHA into Alg hydrogel increase the source of $\mathrm{Ca}^{2+}$ ions into the system, as observed by the released $\mathrm{Ca}^{2+}$ concentration from hydrogels detected by ICP-AES. The $\mathrm{Ca}^{2+}$ ions could affect the remineralization of demineralized areas, since $\mathrm{Ca}^{2+}$ concentration leads to a growth in the saturation of mineralized tissues with nanoHA, favoring the deposition of apatite in the lesions and eventually promoting remineralization [52]. Moreover, the amount of $\mathrm{Ca}^{2+}$ ions released from biomaterials can play a vital role in regulating of the chemotaxis, proliferation and osteogenic differentiation of osteoblast cells [49].

The obtained results showed that the nanoHA modified the physicchemical characteristics of Alg-based hydrogel system. As described, these characteristics could play a vital role in the diffusivity of nutrients, proteins, and metabolites, cell-matrix interactions, chemotaxis, cell proliferation/differentiation, healing and mineralization, highlighting the importance of optimizing the synergism between biomaterials to be addressed in bone tissue regeneration.

The biological response of Alg-nanoHA hydrogel system was also evaluated trough in vitro cytocompatibility assessment with human osteoblastic cells and ex vivo functional assessment of bone formation. In the present study, hydrogels with nanoHA at 30\% promoted higher cell proliferation when compared to those with higher nanoHA content (50 and 70\%), at 14 and 21 days, underlining the importance of nanoHA content for the bioactivity of the composite. Likewise, Eosoly et al. found that MC3T3 cells proliferation was affected when high content of nanoHA was used within PCL composites [21]. One possible explanation is the optimal amount of $\mathrm{Ca}^{2+}$ ions released from composites, due to the solubility of nanoHA and of Alg hydrogel [53,54]. Several authors have described that $\mathrm{Ca}^{2+}$ ions released from composites (e.g. calcium alginate, nanohydroxyapatite-chitosan, polysaccharideenriched calcium sulfate) can affect significantly the metabolism of osteoblast cells and bone remodeling within the implantation site $[28,44,49,55]$. The increase in $\mathrm{Ca}^{2+}$ ions concentration could lead to a disturb within the intracellular calcium homeostasis, which plays a vital role in cell proliferation and apoptosis [56,57], for instance, calcium active calcium-dependent endonucleases that cleavage the DNA $[56,58,59]$, and lead to a decrease of cell proliferation. Some authors pointed out that cell proliferation is hydroxyapatite nanoparticles dosedependent [56,59].

The cell morphology and cytoskeletal organization are important clues of cell homeostasis [60]. Some authors have described that more elongated cells present a higher degree of cytoskeletal tension (F-actin filaments) and differential expression of downstream effectors, such as RhoA and ROCK, which could enhance pathways associated with osteogenesis $[60,57]$. In the present study, regardless of the composition of the composites, hMSCs presented elongated morphology with evident cytoplasmic extensions, indicating good cell functionality and osteogenic potential.

hMSCs cultures grown in the presence of composites were further characterized for the activation of the osteogenic program, with the assessment of Runx2, SP7, Col1a1, and BGLAP gene expression. Runx2 is a key transcriptional regulator for the osteogenic differentiation, 
directly and indirectly modulating the expression of osteoblastic-specific genes; SP7is also a transcription factor that acts downstream of Runx2, being necessary for the later differentiation of the osteogeniccommitted cells into mature osteoblasts [61,62]. Accordingly, hMSCs grown under osteogenic-stimulating conditions (i.e. in the presence of dexamethasone and ascorbic acid) presented significantly higher levels of both Runx2 and Sp7 transcription factors, a process that seems to result from the upregulation of distinct intracellular pathways, namely converging to the TAZ and MPK1 activation [63]. Comparatively, hMSCs cultures grown in the presence of Alg were found to display Runx2 levels similar to basal control, whether cultures established in the presence of Alg-nanoHA hydrogels presented significantly higher levels than basal control, and further found to be similar (Alg-nanoHA70) or significantly higher (Alg-nanoHA30 and Alg-nanoHA50) than those attained in osteogenic-induced hMSCs. This suggests that Alg-nanoHA hydrogels are greatly effective on inducing osteogenesis in vitro through a significant induction of the master osteogenic regulator Runx2 [61]. Assayed hydrogels were also found to induce the expression of Sp7 to levels like to those attained in osteogenic-induced cultures, further sustaining the increased ability to promote the osteoblastic differentiation. Runx2 and Sp7 transcription factors may subsequently regulate the expression of osteoblastic matrix-related genes such as alkaline phosphatase, bone sialoprotein and, as presently evaluated, Colla1 and BGLAP, by binding to specific enhancer regions [64]. Col1a1 is a major constituent of the organic extracellular matrix of bone tissue and its expression has been found to occur at high levels during the early stages of osteogenesis, considering an early osteogenic marker [65]. BGLAP codes a major non-collagenous protein of the extracellular matrix being regarded as a late marker of the osteogenic differentiation pathway with a high-level expression associated with the maturation and late differentiation of osteoblastic populations [65]. BGLAP product further contains a Gla (gamma carboxyglutamate) domain, which binds to calcium and hydroxyapatite, regulating bone mineral maturation [65]. Alg-nanoHA hydrogels, particularly Alg-nanoHA30 and Alg-nanoHA50, were found to significantly increase Col1a1 and BGLAP to levels similar to those attained in osteogenicinduced cultures, sustaining the increased osteogenic activation of hMSCs.

Ex vivo data underlined the in vitro findings, revealing an enhanced osteogenic response by Alg-nanoHA hydrogels, particularly the AlgnanoHA30 composition. This condition induced a strong collagenous matrix deposition at the defect margin and stimulated the mineralized trabecular organization at the collar structure. Compositions with higher content of nanoHA (i.e. Alg-nanoHA50 and Alg-nanoHA70) presented an inferior osteogenic activation, whether Alg hydrogel induced the lowest osteogenic activation, in a way similar to control. Previous studies have demonstrated the osteogenic-inducing ability of chitosan/hydroxyapatite composite systems, in bone tissue-related applications $[20,66,67]$. Notwithstanding, no previous studies have addressed the development and biological evaluation of Alg hydrogels' loading with nanoHA particles at different ratios. In the present study, the ability of Alg-nanoHA composites to significantly induce the osteogenic activation in both in vitro and ex vivo systems was observed. Furthermore, a differential biological response was attained with the loading of distinct nanoHA particles' proportions.

\section{Conclusions}

This work denoted that the nanoHA concentration has a vital role in physic-chemical properties and biological response of the assayed composite. Broadly, physic-chemical features, like water-swelling ratio, stability at extreme $\mathrm{pH}$ conditions, apatite formation and $\mathrm{Ca}^{2+}$ release from Alg-nanoHA hydrogels were nanoHA dose-dependent. The biological response of composites was influenced by nanoHA content, in which Alg-based hydrogel with the nanoHA 30\% content enhanced, in vitro, the osteoblastic cells' proliferation and osteogenic activation, and, in ex vivo, enhanced the collagenous deposition and trabecular bone formation, while those with higher concentrations (50 and 70\%) impaired the biological response. The results showed the need to optimize the ratio of the composite's components in order to maximize bone tissue regeneration.

\section{Acknowledgments}

This work was financed by FEDER - Fundo Europeu de Desenvolvimento Regional funds through the COMPETE 2020 Operacional Programme for Competitiveness and Internationalisation (POCI), Portugal 2020, by Portuguese funds through FCT/MCTES in the framework of the project "institute for Research and Innovation in Health Sciences" (POCI-01-0145-FEDER-007274), by Project Biotherapies (NORTE-01-0145-FEDER-000012) and by Joana Barros' PhD grant (SFRH/BD/102148/2014). The authors would also like to acknowledge Rui Rocha (CEMUP), Rui Fernandes (HEMS), Rossana Correia (HEMS) and Liliana Grenho (FMDUP).

\section{References}

[1] P. Parhi, A. Ramanan, A.R. Ray, Preparation and characterization of alginate and hydroxyapatite-based biocomposite, J. Appl. Polym. Sci. 102 (2006) 5162-5165.

[2] G. Tozzi, A. De Mori, A. Oliveira, M. Roldo, Composite hydrogels for bone regeneration, Materials 9 (2016).

[3] M.C. Birt, D.W. Anderson, E.B. Toby, J.X. Wang, Osteomyelitis: recent advances in pathophysiology and therapeutic strategies, J. Orthop. 14 (2017) 45-52.

[4] X. Zhang, X. Yin, J. Luo, X. Zheng, H. Wang, J. Wang, et al., Novel hierarchical nitrogen-doped multiwalled carbon nanotubes/cellulose/nanohydroxyapatite nanocomposite as an osteoinductive scaffold for enhancing bone regeneration, ACS Biomaterials Science \& Engineering 5 (2019) 294-307.

[5] M. Maisani, S. Ziane, C. Ehret, L. Levesque, R. Siadous, J.F. Le Meins, et al., A new composite hydrogel combining the biological properties of collagen with the mechanical properties of a supramolecular scaffold for bone tissue engineering, J. Tissue Eng. Regen. Med. 12 (2018) e1489-e1500.

[6] S. Obara, T. Yamauchi, N. Tsubokawa, Evaluation of the stimulus response of hydroxyapatite/calcium alginate composite gels, Polym. J. 42 (2010) 161-166.

[7] G. Turco, E. Marsich, F. Bellomo, S. Semeraro, I. Donati, F. Brun, et al., Alginate/ hydroxyapatite biocomposite for bone ingrowth: a trabecular structure with high and isotropic connectivity, Biomacromolecules 10 (2009) 1575-1583.

[8] L. Chen, R. Shen, S. Komasa, Y. Xue, B. Jin, Y. Hou, et al., Drug-loadable calcium alginate hydrogel system for use in oral bone tissue repair, Int. J. Mol. Sci. 18 (2017).

[9] F.O.M.S. Abreu, C. Bianchini, M.M.C. Forte, T.B.L. Kist, Influence of the composition and preparation method on the morphology and swelling behavior of alginatechitosan hydrogels, Carbohyd Polym 74 (2008) 283-289.

[10] B. Sarker, R. Singh, R. Silva, J.A. Roether, J. Kaschta, R. Detsch, et al., Evaluation of fibroblasts adhesion and proliferation on alginate-gelatin crosslinked hydrogel, PLoS One 9 (2014) e107952.

[11] M.O. Dalheim, J. Vanacker, M.A. Najmi, F.L. Aachmann, B.L. Strand, B.E. Christensen, Efficient functionalization of alginate biomaterials, Biomaterials 80 (2016) 146-156.

[12] E.S. Place, L. Rojo, E. Gentleman, J.P. Sardinha, M.M. Stevens, Strontium- and zinc alginate hydrogels for bone tissue engineering, Tissue Eng Part A 17 (2011) 2713-2722.

[13] Y. Luo, A. Lode, C. Wu, J. Chang, M. Gelinsky, Alginate/nanohydroxyapatite scaffolds with designed core/shell structures fabricated by 3D plotting and in situ mineralization for bone tissue engineering, ACS Appl. Mater. Interfaces 7 (2015) 6541-6549.

[14] S. Sancilio, M. Gallorini, C. Di Nisio, E. Marsich, R. Di Pietro, H. Schweikl, et al., Alginate/hydroxyapatite-based nanocomposite scaffolds for bone tissue engineering improve dental pulp biomineralization and differentiation, Stem Cells Int. 2018 (2018) 9643721.

[15] K. Fox, P.A. Tran, N. Tran, Recent advances in research applications of nanophase hydroxyapatite, Chemphyschem 13 (2012) 2495-2506.

[16] J. Luo, X. Zhang, J. Ong'achwa Machuki, C. Dai, Y. Li, K. Guo, et al., Three-dimensionally $\mathrm{N}$-doped graphene-hydroxyapatite/agarose as an osteoinductive scaffold for enhancing bone regeneration, ACS Applied Bio Materials 2 (2019) 299-310.

[17] M.C. Du, W.X. Song, Y. Cui, Y. Yang, J.B. Li, Fabrication and biological application of nano-hydroxyapatite (nHA)/alginate (ALG) hydrogel as scaffolds, J. Mater. Chem. 21 (2011) 2228-2236.

[18] Y. Cai, J. Yu, S.C. Kundu, J. Yao, Multifunctional nano-hydroxyapatite and alginate/gelatin based sticky gel composites for potential bone regeneration, Mater. Chem. Phys. 181 (2016) 227-233.

[19] M. Nabavinia, A.B. Khoshfetrat, H. Naderi-Meshkin, Nano-hydroxyapatite-alginategelatin microcapsule as a potential osteogenic building block for modular bone tissue engineering, Mater. Sci. Eng. C 97 (2019) 67-77.

[20] X.-F. Wang, P.-J. Lu, Y. Song, Y.-C. Sun, Y.-G. Wang, Y. Wang, Nano hydroxyapatite particles promote osteogenesis in a three-dimensional bio-printing construct 
consisting of alginate/gelatin/hASCs, RSC Adv. 6 (2016) 6832-6842.

[21] S. Eosoly, N.E. Vrana, S. Lohfeld, M. Hindie, L. Looney, Interaction of cell culture with composition effects on the mechanical properties of polycaprolactone-hydroxyapatite scaffolds fabricated via selective laser sintering (SLS), Mater. Sci. Eng. C 32 (2012) 2250-2257.

[22] S. Salmasi, L. Nayyer, A.M. Seifalian, G.W. Blunn, Nanohydroxyapatite effect on the degradation, osteoconduction and mechanical properties of polymeric bone tissue engineered scaffolds, Open Orthop J 10 (2016) 900-919.

[23] X. Zhang, W. Chang, P. Lee, Y. Wang, M. Yang, J. Li, et al., Polymer-ceramic spiral structured scaffolds for bone tissue engineering: effect of hydroxyapatite composition on human fetal osteoblasts, PLoS One 9 (2014) e85871.

[24] N. Mohammed, N. Grishkewich, R. Berry, K. Tam, Cellulose nanocrystal-alginate hydrogel beads as novel adsorbents for organic dyes in aqueous solutions, Cellulose 22 (2015) 3725-3738.

[25] T. Kokubo, H. Takadama, How useful is SBF in predicting in vivo bone bioactivity? Biomaterials 27 (2006) 2907-2915.

[26] C.F. dos Santos, P.S. Gomes, M.M. Almeida, M.G. Willinger, R.P. Franke, M.H. Fernandes, et al., Gold-dotted hydroxyapatite nanoparticles as multifunctional platforms for medical applications, RSC Adv. 5 (2015) 69184-69195.

[27] E.L. Smith, J.M. Kanczler, C.A. Roberts, R.O. Oreffo, Developmental cues for bone formation from parathyroid hormone and parathyroid hormone-related protein in an ex vivo organotypic culture system of embryonic chick femora, Tissue Eng Part C Methods 18 (2012) 984-994.

[28] N. Cao, X.B. Chen, D.J. Schreyer, Influence of calcium ions on cell survival and proliferation in the context of an alginate hydrogel, ISRN Chemical Engineering 2012 (2012) 9.

[29] G. Montalbano, S. Toumpaniari, A. Popov, P. Duan, J. Chen, K. Dalgarno, et al. Synthesis of bioinspired collagen/alginate/fibrin based hydrogels for soft tissue engineering, Mater. Sci. Eng. C 91 (2018) 236-246.

[30] Y.-W. Kim, J.E. Kim, Y. Jung, J.-Y. Sun, Non-swellable, cytocompatible pHEMAalginate hydrogels with high stiffness and toughness, Mater. Sci. Eng. C 95 (2019) $86-94$.

[31] S. van Rijt, P. Habibovic, Enhancing regenerative approaches with nanoparticles, J. R. Soc. Interface 14 (2017).

[32] M. Biondi, A. Borzacchiello, L. Mayol, L. Ambrosio, Nanoparticle-integrated hy drogels as multifunctional composite materials for biomedical applications, Gels 1 (2015) 162

[33] S. Pacelli, S. Basu, C. Berkland, J. Wang, A. Paul, Design of a cytocompatible hydrogel coating to modulate properties of ceramic-based scaffolds for bone repair, Cell. Mol. Bioeng. 11 (2018) 211-217.

[34] S. Ahirrao, P. Gide, B. Shrivastav, P. Sharma, Extended release of theophylline through sodium alginate hydrogel beads: effect of glycerol on entrapment efficiency, drug release, Particul Sci Technol 32 (2014) 105-111.

[35] M.J. Costa, A.M. Marques, L.M. Pastrana, J.A. Teixeira, S.M. Sillankorva, M.A. Cerqueira, Physicochemical properties of alginate-based films: effect of ionic crosslinking and mannuronic and guluronic acid ratio, Food Hydrocoll. 81 (2018) 442-448.

[36] M. Jager, H.P. Jennissen, F. Dittrich, A. Fischer, H.L. Kohling, Antimicrobial and osseointegration properties of nanostructured titanium orthopaedic implants, Materials (Basel, Switzerland) (2017) 10.

[37] S.K. Nishimoto, M. Nishimoto, S.W. Park, K.M. Lee, H.S. Kim, J.T. Koh, et al., The effect of titanium surface roughening on protein absorption, cell attachment, and cell spreading, Int. J. Oral Maxillofac. Implants 23 (2008) 675-680.

[38] N. Annabi, J.W. Nichol, X. Zhong, C. Ji, S. Koshy, A. Khademhosseini, et al., Controlling the porosity and microarchitecture of hydrogels for tissue engineering, Tissue Eng. B Rev. 16 (2010) 371-383.

[39] J. Zhang, Q. Wang, A. Wang, In situ generation of sodium alginate/hydroxyapatite nanocomposite beads as drug-controlled release matrices, Acta Biomater. 6 (2010) 445-454.

[40] L. Fan, J.P. Zhang, A.Q. Wang, In situ generation of sodium alginate/hydroxyapatite/halloysite nanotubes nanocomposite hydrogel beads as drug-controlled release matrices, J. Mater. Chem. B 1 (2013) 6261-6270.

[41] M.G. Carneiro-da-Cunha, M.A. Cerqueira, B.W.S. Souza, J.A. Teixeira, A.A. Vicente, Influence of concentration, ionic strength and $\mathrm{pH}$ on zeta potential and mean hydrodynamic diameter of edible polysaccharide solutions envisaged for multinanolayered films production, Carbohyd Polym 85 (2011) 522-528.

[42] V. Uskokovic, R. Odsinada, S. Djordjevic, S. Habelitz, Dynamic light scattering and zeta potential of colloidal mixtures of amelogenin and hydroxyapatite in calcium and phosphate rich ionic milieus, Arch. Oral Biol. 56 (2011) 521-532.

[43] R. Smeets, A. Kolk, M. Gerressen, O. Driemel, O. Maciejewski, B. HermannsSachweh, et al., A new biphasic osteoinductive calcium composite material with a negative zeta potential for bone augmentation, Head Face Med 5 (2009) 13.

[44] Y.M. Chen, J.P. Gong, M. Tanaka, K. Yasuda, S. Yamamoto, M. Shimomura, et al., Tuning of cell proliferation on tough gels by critical charge effect, J. Biomed. Mater. Res. A 88 (2009) 74-83.

[45] D.A. Chakkalakal, A.A. Mashoof, J. Novak, B.S. Strates, M.H. McGuire, Mineralization and $\mathrm{pH}$ relationships in healing skeletal defects grafted with demineralized bone matrix, J. Biomed. Mater. Res. 28 (1994) 1439-1443.

[46] M. Matyash, F. Despang, C. Ikonomidou, M. Gelinsky, Swelling and mechanical properties of alginate hydrogels with respect to promotion of neural growth, Tissue Eng Part C Methods 20 (2014) 401-411.

[47] C.S.D. Lee, H.R. Moyer, R.A. Gittens, J.K. Williams, A.L. Boskey, B.D. Boyan, et al., Regulating in vivo calcification of alginate microbeads, Biomaterials 31 (2010) 4926-4934.

[48] S. Bertazzo, W.F. Zambuzzi, D.D.P. Campos, T.L. Ogeda, C.V. Ferreira, C.A. Bertran, Hydroxyapatite surface solubility and effect on cell adhesion, Colloid Surface B 78
(2010) 177-184.

[49] S. Dhivya, S. Saravanan, T.P. Sastry, N. Selvamurugan, Nanohydroxyapatite-reinforced chitosan composite hydrogel for bone tissue repair in vitro and in vivo, J Nanobiotechnol 13 (2015).

[50] D. Suarez-Gonzalez, K. Barnhart, E. Saito, R. Vanderby, S.J. Hollister, W.L. Murphy, Controlled nucleation of hydroxyapatite on alginate scaffolds for stem cell-based bone tissue engineering, J. Biomed. Mater. Res. A 95a (2010) 222-234.

[51] L. Jongpaiboonkit, T. Franklin-Ford, W.L. Murphy, Growth of hydroxyapatite coatings on biodegradable polymer microspheres, Acs Appl Mater Inter 1 (2009) 1504-1511.

[52] E. Pepla, L.K. Besharat, G. Palaia, G. Tenore, G. Migliau, Nano-hydroxyapatite and its applications in preventive, restorative and regenerative dentistry: a review of literature, Ann Stomatol (Roma) 5 (2014) 108-114.

[53] K.Y. Lee, D.J. Mooney, Alginate: properties and biomedical applications, Prog. Polym. Sci. 37 (2012) 106-126.

[54] Z.F. Chen, B.W. Darvell, V.W. Leung, Hydroxyapatite solubility in simple inorganic solutions, Arch. Oral Biol. 49 (2004) 359-367.

[55] G. Chan, D.J. Mooney, $\mathrm{Ca}(2+)$ released from calcium alginate gels can promote inflammatory responses in vitro and in vivo, Acta Biomater. 9 (2013) 9281-9291.

[56] R. Meena, K.K. Kesari, M. Rani, R. Paulraj, Effects of hydroxyapatite nanoparticles on proliferation and apoptosis of human breast cancer cells (MCF-7), J. Nanopart. Res. 14 (2012) 712.

[57] R. Zhao, P. Xie, K. Zhang, Z. Tang, X. Chen, X. Zhu, et al., Selective effect of hydroxyapatite nanoparticles on osteoporotic and healthy bone formation correlates with intracellular calcium homeostasis regulation, Acta Biomater. 59 (2017) 338-350.

[58] J. Zhang, X. Luo, D. Barbieri, A.M. Barradas, J.D. de Bruijn, C.A. van Blitterswijk, et al., The size of surface microstructures as an osteogenic factor in calcium phosphate ceramics, Acta Biomater. 10 (2014) 3254-3263.

[59] W. Tang, Y. Yuan, C. Liu, Y. Wu, X. Lu, J. Qian, Differential cytotoxicity and particle action of hydroxyapatite nanoparticles in human cancer cells, Nanomedicine (London, England) 9 (2014) 397-412.

[60] P.S. Mathieu, E.G. Loboa, Cytoskeletal and focal adhesion influences on mesenchymal stem cell shape, mechanical properties, and differentiation down osteogenic adipogenic, and chondrogenic pathways, Tissue Eng. B Rev. 18 (2012) 436-444.

[61] E.D. Jensen, R. Gopalakrishnan, J.J. Westendorf, Regulation of gene expression in osteoblasts, BioFactors (Oxford, England) 36 (2010) 25-32.

[62] T. Komori, Regulation of bone development and extracellular matrix protein genes by RUNX2, Cell Tissue Res. 339 (2010) 189-195.

[63] F. Langenbach, J. Handschel, Effects of dexamethasone, ascorbic acid and B-glycerophosphate on the osteogenic differentiation of stem cells in vitro, Stem Cell Res Ther 4 (2013) 117.

[64] H. Harada, S. Tagashira, M. Fujiwara, S. Ogawa, T. Katsumata, A. Yamaguchi, et al., Cbfa1 isoforms exert functional differences in osteoblast differentiation, J. Biol. Chem. 274 (1999) 6972-6978.

[65] G. Karsenty, Minireview: transcriptional control of osteoblast differentiation, Endocrinology 142 (2001) 2731-2733.

[66] Y. He, Y. Dong, F. Cui, X. Chen, R. Lin, Ectopic osteogenesis and scaffold biodegradation of nano-hydroxyapatite-chitosan in a rat model, PLoS One 10 (2015) e0135366.

[67] E. Chatzipetros, P. Christopoulos, C. Donta, K.I. Tosios, E. Tsiambas, D. Tsiourvas, et al., Application of nano-hydroxyapatite/chitosan scaffolds on rat calvarial critical-sized defects: a pilot study, Medicina oral, patologia oral y cirugia bucal 23 (2018) e625-e632.

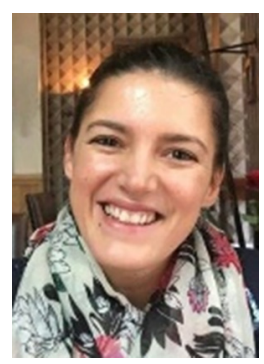

$\mathbf{J}$ Barros has a degree in Genetics and Biotechnology and a master's degree in Biotechnology for Health Sciences at UTAD. In 2011, she started her research activities at INEB, in the NanoBiofilm project - "Nanohydroxyapatite antibacterial surfaces to prevent biofilm-associated bone infection". From 2014 to 2015, she worked as research assistant fellowship in European project "SUSCLEAN Sustainable Cleaning and Disinfection in Fresh-Cut Food industries", at FEUP. Since 2015, she is $\mathrm{PhD}$ student in Biomedical Engineering at i3S, INEB, and FEUP. She is author of 12 articles in international peer-reviewed journals. 


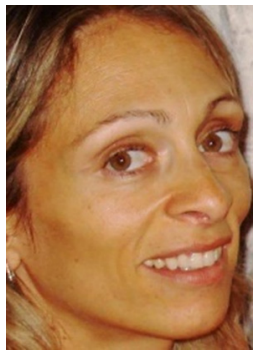

MP Ferraz has a Ph.D. in Sciences of the Engineering at the University of Porto, and she is Associate Professor at University Fernando Pessoa, Porto, Portugal. She has $>200$ collaborators as co-authorship of scientific works and participated in 10 national projects. She has supervised 2 completed Ph.D. theses and published 60 international peer-reviewed papers. The major research interests are focused on Medical Sciences with emphasis on Health Sciences and Engineering and Technology, mainly on the development of biomaterials for bone tissue regeneration, and as drug delivery systems for the control of bone infections.

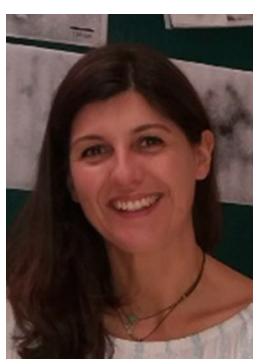

J Azeredo has a Ph.D. in Chemical Sciences at the University of Minho, and she is Associate Professor at U. Minho, Braga, Portugal. She has been responsible for 2 European projects and 7 national projects. She has supervised 15 completed Ph.D. theses and published 150 international peer-reviewed papers. Health Biotechnology and Bioengineering is her research line, addressing themes such as phage technology, characterization of the interaction of phages with biofilms, development of biomaterials with phages incorporated for treatment of infectious disease.

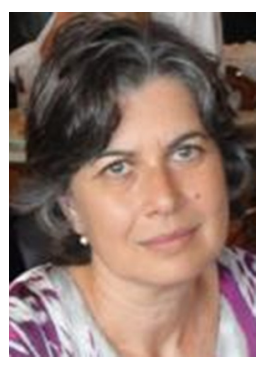

MH Fernandes got her Ph.D. in Organic Chemistry and Pharmacology at Imperial College of Science and Technology, University of London, UK. She is Full Professor at the Faculty of Dentistry, U. Porto, Portugal, and she is the Principal Investigator of the Laboratory for Bone Metabolism and Regeneration. She has authored $>150$ manuscripts, with research focused in the (i) bone metabolism and regeneration, (ii) regeneration of soft and hard oral tissues, and (iii) cytotoxicity and biocompatibility studies - following in vitro, ex vivo and in vivo characterization, in a multidisciplinary research network.

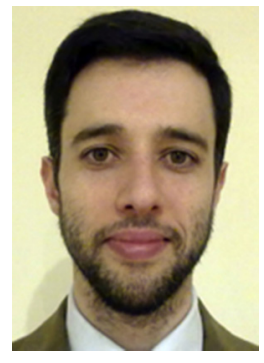

PS Gomes has a Ph.D. in Dental Medicine, and he is Associate Professor at the Faculty of Dentistry, U. Porto, Portugal. He participated in 11 national projects and has supervised completed 25 master's dissertations. He published 89 international peer-reviewed papers. His interest's areas cover cytotoxicity and biocompatibility studies through in vitro, ex vivo and in vivo characterization, pharmacological approaches for the enhancement of bone regeneration and biological response to biomaterials and medical devices for bone tissue applications.

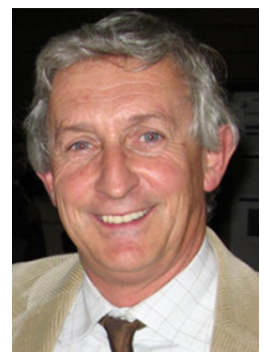

FJ Monteiro has a Ph.D. in Materials Engineering and he is a Full Professor at the University of Porto, where he is head of Doctoral Program in Biomedical Engineering. He is also a senior researcher at the Institute of Research and Innovation in Health and Group Leader of Biocomposites group in the same institute and has authored $>150$ manuscripts. His areas of scientific activity include nanophased ceramic/polymer composite for tissue regeneration and drug releasing, controlled surfaces for guided tissue regeneration, antibacterial biomaterials, biomarkers for cell targeting, protein interaction with biomaterials and development of liquid biopsy devices. 